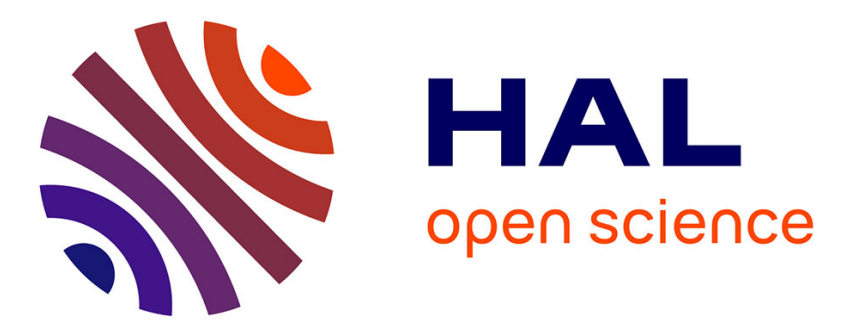

\title{
Maintenance Decision-Making for Systems Operating Under Indirect Condition Monitoring: Value of Online Information and Impact of Measurement Uncertainty
}

Khac Tuan Huynh, Anne Barros, Christophe Bérenguer

\section{- To cite this version:}

Khac Tuan Huynh, Anne Barros, Christophe Bérenguer. Maintenance Decision-Making for Systems Operating Under Indirect Condition Monitoring: Value of Online Information and Impact of Measurement Uncertainty. IEEE Transactions on Reliability, 2012, 61 (2), pp.410-425. 10.1109/TR.2012.2194174 . hal-00790730

\author{
HAL Id: hal-00790730 \\ https://hal.science/hal-00790730
}

Submitted on 21 Feb 2013

HAL is a multi-disciplinary open access archive for the deposit and dissemination of scientific research documents, whether they are published or not. The documents may come from teaching and research institutions in France or abroad, or from public or private research centers.
L'archive ouverte pluridisciplinaire HAL, est destinée au dépôt et à la diffusion de documents scientifiques de niveau recherche, publiés ou non, émanant des établissements d'enseignement et de recherche français ou étrangers, des laboratoires publics ou privés. 


\title{
Maintenance Decision-making for Systems Operating Under Indirect Condition Monitoring: Value of Online Information and Impact of Measurement Uncertainty
}

\author{
K.T. Huynh ${ }^{\mathrm{a}}$, A. Barros ${ }^{\mathrm{a}}$, C. Bérenguer ${ }^{\mathrm{a}, \mathrm{b}}$ \\ ${ }^{a}$ Université de technologie de Troyes - Institut Charles Delaunay and STMR UMR CNRS 6279 - 12, rue Marie Curie, \\ BP2060, 10010 Troyes cedex - France \\ ${ }^{b}$ Gipsa-lab ; CNRS ; Grenoble Institute of Technology - 11, rue des Mathématiques - BP46 - 38402 Saint Martin d'Hères \\ cedex - France
}

\begin{abstract}
This paper deals with maintenance decision-making for single-unit deteriorating systems operating under indirect condition monitoring. Based on a degradation and measurement model of crack growth propagation, two new maintenance policies using prognostic information are introduced. Their maintenance cost models are evaluated jointly by analytical and simulation approaches, and are compared with two more classical benchmark models. Such complete models integrating degradation phenomenon, monitoring characteristics, state estimation, prognostics, and maintenance assessment can give rise to fruitful numerical analyses and discussions.

The main contributions of the paper are to $i$ ) analyze jointly the condition-based and dynamic structure of the considered maintenance policies; $i i)$ propose some effective methods to reduce the effect of measurement uncertainty in condition-based maintenance decision-making; and $\mathrm{iii}$ ) show the relevance of quantification methods when deciding to resort to prognostic approaches, and to invest in condition monitoring devices.
\end{abstract}

Index Terms - Degradation, indirect condition monitoring, maintenance policy, measurement uncertainty, particle filter, prognostic, real-time estimation.

\section{Acronyms}

CBM condition-based maintenance

TBM time-based maintenance

BR block replacement

PIR periodic inspection and replacement

QIR quantile-based inspection and replacement

DR dynamic replacement

PF particle filter or particle filtering 


\section{Notations}

$C, n, \beta$

$\beta_{0}, \beta_{1}$

$x_{t_{i}}, z_{t_{i}}$

$\omega_{t_{i}}, v_{t_{i}}$

$\sigma_{\omega}, \sigma_{v}$

$\Delta t, \Delta x_{t_{i}}$ $f(\cdot), f(\cdot \mid \cdot)$

$d, M$

$T_{d}, T_{M}$ $C_{i}, C_{d}, C_{p}, C_{c}$

$C(\cdot)$

$C^{\infty}$

$C_{D}^{\infty}, C_{Q}^{\infty}$

$S, S^{(n)}$

$S_{D}^{(n)}, S_{Q}^{(n)}$

$N_{h}, N_{s}$

$T, \alpha$

$T_{i}, T_{\text {pred }}^{(i)}$

$\mathbf{Z}_{T_{0: i}}$

$\widehat{x}_{t_{i}}^{(k)}, \widetilde{x}_{t_{i}}^{(k)}, w_{i}^{(k)}$

$\widehat{x}_{t_{i}}, \widehat{f}(\cdot \mid \cdot)$

$C_{\text {pred }}(\cdot)$

$R(\cdot)$

$P_{p, D}, P_{p, Q}$

$N_{i, D}, N_{i, Q}$

$W_{d, D}, W_{d, Q}$ constant parameters in the Paris-Erdogan crack growth model

constant parameters in measurement logit model

true state, and measured state at time $t_{i}$

system noise, and measurement noise at time $t_{i}$

standard derivation of system noise, and measurement noise

discretization time step $t_{i+1}=t_{i}+\Delta t$, degradation rate $\Delta x_{t_{i}}=\left(x_{t_{i}}-x_{t_{i-1}}\right) / \Delta t$

unconditional, and conditional probability density function

critical material thickness, and preventive replacement degradation threshold

failure time, and hitting time of threshold $M$

inspection cost, system inactivity cost rate, preventive replacement cost, and corrective replacement cost

accumulated maintenance cost

expected maintenance cost rate

expected maintenance cost rate of DR policy, and QIR policy

renewal (replacement) cycle, and length of the first renewal cycle of the $n$-th history length of the first renewal cycle of $n$-th history under DR policy, and QIR policy number of simulation histories, and number of sampled particles

periodic inter-inspection interval, and quantile level

$i$-th inspection time, and predictive replacement time according to $T_{i}$

sequence of measurements at inspection time up to $T_{i}$

estimated state, predictive state, and importance weight at $t_{i}$ for the $k$-th particle

estimated state at $t_{i}$, and estimated conditional probability density function

cost criterion to optimize in real time the predictive replacement time in the DR policy conditional reliability to optimize in real time the inspection time in the QIR policy preventive replacement probability in a renewal cycle under the DR policy, and the QIR policy

inspection number effected in a renewal cycle under the DR policy, and the QIR policy downtime interval in a renewal cycle under the DR policy, and the QIR policy 


\section{Introduction}

With the dissemination of condition monitoring techniques, Condition-Based Maintenance (CBM) is considered to be a promising approach to improve the durability, reliability, and maintainability of industrial systems. Many CBM models [1], [2], [3], [4] have been developed. Among the existing maintenance models, a very basic analysis might state that the "best" maintenance decision rules have to take into account all the available online information, have to be condition-based (i.e., the decision relies on an indicator of the system state), and dynamic (i.e., the structure of the decision rule itself can adapt to the condition information). In this paper, we deal with a more precise analysis: even if online condition information is available, it is not always clear whether condition-based and dynamic (or predictive) maintenance decision rules are always relevant. The answer is obviously logically dependent on the quality of the monitoring information, the structure of the maintenance decision rule, the dynamic characteristic of system evolution, as well as on the maintenance costs.

In the literature, very few models take into full account all these aspects. For example, some recent models propose to quantify the performance of dynamic maintenance rules [5], [6] based on perfect condition information which corresponds exactly to the real system state. These considerations seem to be less practical because the condition monitoring data, which is usually obtained through indirect inspection techniques [7], are always affected to some degree by noise and disturbances. Dealing with more practical approaches, some studies (e.g., as in [8], [9], and [10]) are devoted to the quality of the condition information, and propose methods (see e.g., the review [11]) for inferring the underlying system state from noisy monitoring data. However, the associated maintenance models in these works are quite simple, and do not reflect the dynamic characteristic of the decision rule. Finally, the system state evolution is often considered to be stationary with no influencing factors (e.g., the system state evolution is modeled by a homogeneous gamma process [12], [13]). This state is not the best test case to assess the performance of dynamic maintenance, which can adapt to an acceleration of the degradation rate, for example. In this context, our aim is to base our work on existing degradation models and classical simulation techniques to study jointly three main problems in maintenance decision-making: i) the non-stationary aspect of the degradation phenomenon, ii) the imperfect nature of condition monitoring information, and iii) the condition-based or dynamic structure of the maintenance policies. Hence, we break out of classical assumptions of maintenance models that allow complete analytical maintenance rule optimization.

As a test case, we focus in this paper on the fatigue crack growth degradation phenomenon. The classical non-destructive ultrasonic techniques are used to inspect the system, so the crack depth is not 
directly observable. Hence a degradation and measurement model is necessary to describe both the system characteristics and the indirect monitoring. Such a model is proposed in [14], and is reused here. It is based on a non-stationary Markovian process for the degradation phenomenon, and a logit model for the monitoring. Based on this model, two ways for integrating the online information in maintenance decisions are introduced through a prediction of the system evolution (real-time prognostic). In one case, the decision rule is non-condition-based and dynamic (the Dynamic Replacement (DR) policy); in the other case, it is condition-based and dynamic (the Quantile-based Inspection and Replacement (QIR) policy). The performance of these decision rules is assessed through the optimal expected maintenance cost rate by comparing with two more classical maintenance policies [15]: one is only condition-based (the Periodic Inspection and Replacement (PIR) policy), and the other one is systematic, i.e., only based on the time (the Block Replacement (BR) policy). The cost models of these policies are developed, and jointly evaluated by both analytical and simulation approaches.

Regarding the analytical approach, we calculate the conditional probabilities of maintenance events for policy optimization. Even if analytical developments are not complete due to the complexity of the degradation, monitoring, and maintenance models, we have a theoretical framework that allows us to determine at which step we have to begin with simulation, and which quantities will be calculated by simulation.

Regarding the simulation approach, we adapt existing simulation methods to calculate the quantities that cannot be derived from the analytical approach. In particular, we apply particle filter techniques, and Monte Carlo simulations (used in [16], [17], [18], [19], [20], [21], [22] for fault diagnostic and prognostic purposes) in the larger framework of maintenance decision-making and performance evaluation.

The main contributions of our paper are as follows.

- Analyzing jointly the condition-based and dynamic structure of new maintenance decision rules (DR policy and QIR policy). This point has not been studied before in the literature.

- Progressing knowledge about the impact of condition monitoring information uncertainty in maintenance decision-making, and showing some methods to limit the negative impact. This study concludes the work on the performance and on the choice of a CBM policy or a Time-based Maintenance (TBM) policy in [23], [15] for the case of imperfect condition monitoring data. Although the measurement uncertainty reduces the performance of the CBM policy, it is still more profitable than the TBM policy for a reasonable level of condition monitoring errors. Furthermore, we show that a dynamic maintenance structure, and a decision based on the estimated degradation constructed from the measurement data, are the effective approaches to limit the negative impact 
of measurement uncertainty.

- Quantifying the value of prognostic information in maintenance decision-making, and assessing the performance of the dynamic maintenance policy. Many authors argue that implementing prognostics approaches in maintenance decision-making can lead to good performance, but none of them has proposed a concrete maintenance model and indicated the appropriate configuration for its application. Through the comparison results of four types of maintenance policies under different configurations of intervention costs and system characteristics, we develop these points. In fact, a dynamic maintenance policy based on prognostic information can be, but not always is, more profitable than a static one. So, a quantification model should be used to justify a choice of a type of maintenance policy.

The remainder of this paper is organized as follows. Section 2 of the paper is devoted to degradation and measurement models. Section 3 deals with a detailed theoretical description of the maintenance models including the maintenance assumptions, the criterion to evaluate the policies' performance, the proposed maintenance policies, as well as the condition-based aspect and dynamic aspect of each policy. In Section 4, a Particle Filtering (PF) procedure, and a Monte Carlo simulation approach are introduced to give the numerical solution for real-time system state estimation and prognostics from noisy measured data, and for the maintenance models assessment. The impact of the uncertainty of measured information, and the value of applying a real-time prognostic for maintenance decision-making, are respectively investigated in Sections 5, and 6. The paper will be closed with some conclusions and directions for future works.

\section{Degradation and measurement model}

Let us consider the well known fatigue crack growth degradation phenomenon, and the associated degradation and measurement model proposed in [14]. The crack depth evolution is modeled by a nonstationary Markovian process, and is assumed to be unobservable directly but can be measured through a non-destructive ultrasonic logit model. A system failure occurs when the crack depth exceeds a critical material thickness $d$.

\subsection{Degradation model}

Degradation modeling of mechanical equipment usually starts with a deterministic description of the underlying degradation phenomenon through physics or chemistry, then randomness can be incorporated into the model to preserve the stochastic nature of a degradation process. In this work, the basis of the 
degradation model is the continuous time, deterministic Paris-Erdogan model which reflects the physical interpretation of the phenomenon

$$
\frac{d x}{d t}=C(\Delta K)^{n}
$$

where $x$ is the crack depth, $t$ is the time, $\Delta K=\Delta K(x)$ is the stress intensity amplitude, and $C$ and $n$ are constant parameters related to the component material properties [24]. The parameters of the Paris-Erdogan model can be estimated from measured crack growth data [25]. The stress intensity factor is computed by $\Delta K=\beta \sqrt{x}$, where the parameter $\beta$ is related to the applied stress and geometrical configuration of the structure [26].

To preserve the intrinsic randomness of the degradation phenomenon, the aforementioned deterministic Paris-Erdogan crack growth model is randomized. It has been suggested to randomize the deterministic crack propagation by a stochastic process [27], or by a randomization of characteristic parameters (i.e., $C, n$, or $\beta$ ) of the deterministic model [28]. The randomization approach we use here is a special case of Yang's power law model [29] where a lognormal distributed random variable is introduced. The versatility of this randomization approach has been analyzed in [30], and justified on the basis of empirical data in [31], [32]. Consequently, for a time step $\Delta t$ sufficiently small, one can discretize and insert the randomness into (1) as follows [14]

$$
x_{t_{i}}=x_{t_{i-1}}+\mathrm{e}^{\omega_{t_{i}}} C\left(\beta \sqrt{x_{t_{i-1}}}\right)^{n} \Delta t
$$

where $\omega_{t_{i}} \sim \mathcal{N}\left(0, \sigma_{\omega}^{2}\right), i=1,2, \ldots$ are Gaussian random disturbance, $0<x_{t_{i-1}}<x_{t_{i}}<+\infty$. The discretized model allows the recursive calculation of the crack depth $x_{t_{i}}$ at each time step $t_{i}=i \Delta t$ given the crack depth $x_{t_{i-1}}$ at time step $t_{i-1}$, and the parameters of model. Because the actual state $x_{t_{i}}$ depends

only on the last previous state $x_{t_{i-1}},(2)$ represents a non-linear Markov process with conditionally $s$ independent but non-stationary increments whose degradation rate $\Delta x_{t_{i}}=\left(x_{t_{i}}-x_{t_{i-1}}\right) / \Delta t$ follows a lognormal distribution

$$
\Delta x_{t_{i}} \sim \log \mathcal{N}\left(\ln \left(C \cdot\left(\beta \sqrt{x_{t_{i-1}}}\right)^{n}\right), \sigma_{\omega}^{2}\right)
$$

As such, the expected value, and variance of crack depth increment per unit of time are respectively $E\left[\Delta x_{t_{i}}\right]=C \cdot\left(\beta \sqrt{x_{t_{i-1}}}\right)^{n} \mathrm{e}^{\frac{\sigma_{\omega}^{2}}{2}}$, and $\operatorname{var}\left[\Delta x_{t_{i}}\right]=E^{2}\left[\Delta x_{t_{i}}\right] \cdot\left(\mathrm{e}^{\sigma_{\omega}^{2}}-1\right)$.

\subsection{Measurement model}

The crack depth $x_{t_{i}}$ at time step $t_{i}$ is not directly observable, but can be assessed via noisy measured condition information data. We propose to use a classical non-destructive ultrasonic inspection and 
measurement technique whose associated observation $z_{t_{i}}$ is described by the logit model [33]

$$
\ln \frac{z_{t_{i}}}{d-z_{t_{i}}}=\beta_{0}+\beta_{1} \ln \frac{x_{t_{i}}}{d-x_{t_{i}}}+v_{t_{i}}
$$

where $d$ is the critical material thickness of the component, $\beta_{0} \in \mathbb{R}$ and $\beta_{1}>0$ are parameters to be estimated from experimental data, and $v_{t_{i}}$ is a white Gaussian noise such that $v_{t_{i}} \sim \mathcal{N}\left(0, \sigma_{v}^{2}\right)$. This measurement model is valid for all $x_{t_{i}} \in(0, d)$ because, when $x_{t_{i}} \geq d$, then a failure occurs, and no inspection action is carried out because the failure is self-announcing. The measurement variable $z_{t_{i}}$ is thereby a random variable which is defined in the domain $0<z_{t_{i}}<d$ for all $x_{t_{i}} \in(0, d)$. From (4), given $x_{t_{i}} \in(0, d)$, the likelihood pdf of the measurement $z_{t_{i}}$, which is used to update the importance weights in the PF technique, is given by [18]

$$
f\left(z_{t_{i}} \mid x_{t_{i}}\right)=\frac{1}{\sqrt{2 \pi \sigma_{v}^{2}}} \mathrm{e}^{-\frac{1}{2 \sigma_{v}^{2}}\left(\ln \frac{z_{t_{i}}}{d-z_{t_{i}}}-\beta_{0}-\beta_{1} \ln \frac{x_{t_{i}}}{d-x_{t_{i}}}\right)^{2}} \frac{d}{z_{t_{i}}\left(d-z_{t_{i}}\right)} \cdot 1_{\left\{0<z_{t_{i}}<d\right\}}
$$

where $1_{\{\}}$denotes the indicator function which equals 1 if the argument is true, and 0 otherwise.

\section{Theoretical development of maintenance models}

Under the framework of the degradation and measurement model considered in Section 2, we introduce in this section two decision rules that incorporate the real-time prognostic information in two different ways through a DR policy, and a QIR policy. Because the condition monitoring information collected through indirect measurement is affected by noise and disturbances, the decision rules rely on the estimated degradation state reconstructed from the sequence of measurements, instead of using directly the monitoring information. To assess the performance of these new maintenance policies, the BR policy, and the PIR policy are introduced as benchmarks [15]. Based on the considered maintenance policies, we analyze in detail the nature of the maintenance decision rule to enhance the condition-based and dynamic aspects. Notice that the condition-based aspect refers to decision rules based on an indicator of the actual system state, while the dynamic aspect refers to decision rules where the structure itself can adapt to the condition information. We preliminarily develop the mathematical cost models of the DR policy, and the QIR policy; and indicate the reasons for which these cost models cannot be completely evaluated by an analytical approach, hence numerical methods have to be used to overcome this problem. 


\subsection{Assumptions, and objective cost function}

We assume that the system degradation (i.e., the crack depth) is hidden, so monitoring is then required to reveal the degradation level. Continuous monitoring performed at each time step $\Delta t$ is usually very costly, and sometimes impossible in practical engineering applications. In this framework, it is more suitable to implement discrete monitoring whose length between two successive inspections is a multiple of $\Delta t$. An indirect non-destructive ultrasonic technique is used to inspect the system, so the measurement $Z_{T_{i}}$ is imperfect and instantaneously collected at each discrete inspection time $T_{i}$ with a cost $C_{i}$. Moreover, because the system stops working whenever it fails, one can recognize its failure state without any inspection (i.e., self-announcing failure).

Two maintenance operations are available on the system: a preventive replacement with $\operatorname{cost} C_{p}>C_{i}$, and a corrective replacement with cost $C_{c}$. Because maintenance operations are true physical replacements such that the system is as-good-as-new, the intervention takes negligible time, and the costs of preventive and corrective replacement are fixed for varied degradation levels of the system. Even though both preventive and corrective maintenance actions put the system back in the as-good-as-new state, they are not necessarily identical in any sense in practice because the corrective replacement is unplanned, it has to be performed on a more deteriorated system, and the cost $C_{c}$ can also comprise different costs associated with failure. It is thus likely to be more complex, and more expensive (i.e., $\left.C_{c}>C_{p}\right)$. A replacement, whether preventive or corrective, can only be instantaneously performed at discrete predetermined times (i.e., inspection time, or predictive replacement time). Therefore, there exists a system inactivity after failure, and an additional cost is incurred from the failure time until the next replacement time at a cost rate $C_{d}$.

The aforementioned assumptions are particularly suitable for systems located far from the maintenance crew (e.g., "offshore" systems, hydroelectric or petroleum discharge systems, etc.). Continuous inspection for such systems is almost impossible, and any intervention (i.e., inspection or replacement) is planned and carried out only at discrete prefixed times. Moreover, even if the system failure is selfannouncing, we do not know immediately the failure time, and hence do not provide any intervention. Once the maintenance crew arrives to check the system condition, if the system has already failed, the crew fixes immediately the failure without inspection.

To assess the performance of a maintenance policy, we focus on a widely used criterion which is the expected maintenance cost per unit over an infinite time span. The assumption of an as-good-asnew maintained system leads to the use of the regenerative properties of a system state to compute 
analytically the cost rate [34]

$$
C^{\infty}=\lim _{t \rightarrow \infty} \frac{C(t)}{t}=\frac{E[C(S)]}{E[S]}
$$

where $C(t)$ is the cumulated maintenance cost at time $t$, and $S$ is the time between two successive renewals.

\subsection{Dynamic Replacement model, DR}

\subsubsection{Decision rule}

The main idea of the DR policy is to begin with a classical PIR policy [15] whose intervention (i.e., inspection, replacement) is only possible at periodic prefixed times $T_{i}=i T$, where $i=1,2 \ldots$, and $T$ is called the inter-inspection time interval, which is a multiple value of $\Delta t$. A simple way to introduce dynamic aspects is to allow replacements between $T_{i}$ and $T_{i+1}$ that can be dynamically conditioned by the sequence of measurements up to inspection time $T_{i}, \mathbf{Z}_{T_{0: i}}=\left\{z_{T_{0}}, z_{T_{1}}, \ldots, z_{T_{i}}\right\}, T_{0}=0$. This predictive replacement time (noted $T_{\text {pred }}^{(i)}$ associated with the $i$-th inspection) forms the dynamic aspect of the maintenance policy. The structure of such a decision rule in a renewal cycle can be described by the following scheme.

1. The system condition is periodically inspected at time $T_{i}=i T$ (the first inspection since the last renewal is $\left.T_{1}=T\right)$.

2. If the system is failed at time $T_{i}$, then it is correctively replaced by a new one, and a new renewal cycle starts.

3. If the system is still running at time $T_{i}$, then nothing is done at this time, and a dynamic intervention date $T_{p r e d}^{(i)}$ is optimized given the measurements $\mathbf{Z}_{T_{0: i}}$. If $T_{p r e d}^{(i)}<T_{i+1}$, the system is replaced without additional inspection at time $T_{\text {pred }}^{(i)}$, either preventively if it is still running, or correctively if it has already failed, and a new renewal cycle starts. Otherwise, nothing is done at $T_{\text {pred }}^{(i)}$, and the decision is postponed until the next inspection planed at $T_{i+1}=(i+1) T$.

Such a dynamic decision rule, which allows interventions before $T_{i+1}$ when the risk of failure is high, leads to a more timely maintenance decision; hence, it guarantees the system availability. The predictive replacement time $T_{p r e d}^{(i)}$, and the inter-inspection time interval $T$, are the two decision variables of the DR policy. We can see in Sections 3.2.2 and 3.2.3 that $T_{\text {pred }}^{(i)}$ is dynamically optimized in real-time at each time $T_{i}$ thanks to a cost criterion $C_{\text {pred }}$ calculated over the time interval from the last renewal until a replacement, and $T$ is optimized with $C^{\infty}$ given from (6). 


\subsubsection{Predictive replacement time estimation: real-time optimization of $T_{\text {pred }}^{(i)}$}

The aim of this section is to propose a cost model to dynamically optimize the predictive replacement time $T_{\text {pred }}^{(i)}$ at each inspection time $T_{i}$, given $\mathbf{Z}_{T_{0: i}}$, and the system is still running at $T_{i}$. The basic idea of a dynamic structure is inspired by the works of Cadini et al. in [19]; however, another cost model for optimization of $T_{\text {pred }}^{(i)}$ is used here to accord with the decision rule and the assumptions of the DR policy. Under the considered policy, the inter-inspection time interval $T$ is a decision variable to be optimized. Obviously, the inspection cost influences the length of $T$, and hence the predictive replacement time $T_{\text {pred }}^{(i)}$; thus, it cannot be negligible in the cost criterion to optimize $T_{\text {pred }}^{(i)}$. To do so, we compute the expected maintenance cost rate to optimize $T_{\text {pred }}^{(i)}$ over the entire renewal cycles of the system. And because the future measurements have obviously not yet been collected from the last inspection at $T_{i}$ (the $i$-th inspection since the last renewal time), we use a decision rule similar to the decision of the BR policy [15]. At time $T_{i}+l \Delta t$ in the future, the system is replaced, either preventively if it is still running, or correctively if it will have failed. As such, besides the preventive and corrective replacement costs, the costs incurred for inspection from the last system renewal until the current inspection $T_{i}$ have to be included in the model. Moreover, the cost rate of the system inactivity is also taken into account because the system is not immediately replaced after failure. Thence, the cost criterion to optimize the dynamic time for system replacement $T_{p r e d}^{(i)}$ can be expressed by

$$
\begin{aligned}
C_{\text {pred }}\left(T_{i}+l \Delta t, T, \mathbf{Z}_{T_{0: i}}\right)= & \frac{i C_{i}+C_{p} R\left(T_{i}+l \Delta t, \mathbf{Z}_{T_{0: i}}\right)}{T_{i}+l \Delta t}+ \\
& \frac{C_{c}\left(1-R\left(T_{i}+l \Delta t, \mathbf{Z}_{T_{0: i}}\right)\right)+C_{d} E\left[W_{d}\left(T_{i}+l \Delta t\right)\right]}{T_{i}+l \Delta t}
\end{aligned}
$$

where $l \Delta t, l=1,2, \ldots$, is the residual life defined from the current inspection time $T_{i}$ to the predictive replacement time $T_{i}+l \Delta t, E\left[W_{d}\left(T_{i}+l \Delta t\right)\right]$ is expected downtime interval in the time interval $\left(T_{i}, T_{i}+l \Delta t\right]$, and $R\left(T_{i}+l \Delta t, \mathbf{Z}_{T_{0: i}}\right)$ denotes the conditional reliability of the system at time $T_{i}+l \Delta t$, $l \geq 1$, given the system is still running at $T_{i}$ and $\mathbf{Z}_{T_{0: i}}$. The relevance of the cost criterion (7) is also strengthened by Wang [35]. $E\left[W_{d}\left(T_{i}+l \Delta t\right)\right]$ can be approximated by

$$
E\left[W_{d}\left(T_{i}+l \Delta t\right)\right] \simeq \Delta t\left(l-\sum_{j=1}^{l} R\left(T_{i}+j \Delta t, \mathbf{Z}_{T_{0: i}}\right)\right)
$$

The conditional reliability $R\left(T_{i}+j \Delta t, \mathbf{Z}_{T_{0: i}}\right)$ can be expressed by

$$
R\left(T_{i}+j \Delta t, \mathbf{Z}_{T_{0: i}}\right)=\operatorname{Pr}\left\{x_{T_{i}+j \Delta t}<d \mid x_{T_{i}}<d, \mathbf{Z}_{T_{0: i}}\right\}=\frac{\operatorname{Pr}\left\{x_{T_{i}+j \Delta t}<d \mid \mathbf{Z}_{T_{0: i}}\right\}}{\operatorname{Pr}\left\{x_{T_{i}}<d \mid \mathbf{Z}_{T_{0: i}}\right\}}
$$


To evaluate analytically $R\left(T_{i}+j \Delta t, \mathbf{Z}_{T_{0: i}}\right)$, we need the mathematical formulas of distribution functions of $x_{T_{i}+j \Delta t}$, and $x_{T_{i}}$. Nevertheless, the analytical evaluation of these quantities is not trivial due to the complexity of the considered degradation and measurement model. We will see in the following that the $\mathrm{PF}$ technique is used to provide an empirical approximation of $R\left(T_{i}+l \Delta t, \mathbf{Z}_{T_{0: i}}\right)$.

Consequently, among all future time steps $T_{i}+l \Delta t, l \geq 1$, the suitable predictive replacement time $T_{\text {pred }}^{(i)}$ is the one which minimizes the cost criterion (7)

$$
C_{\text {pred }}\left(T_{\text {pred }}^{(i)}, T, \mathbf{Z}_{T_{0: i}}\right)=\min _{l}\left\{C_{\text {pred }}\left(T_{i}+l \Delta t, T, \mathbf{Z}_{T_{0: i}}\right), l>0\right\}
$$

Such a predictive replacement time $T_{\text {pred }}^{(i)}$ depends on the predetermined inter-inspection time interval $T$, and the sequence of random measurements $\mathbf{Z}_{T_{0: i}}$. Equation (10) means that if no more information about the system state is available, $T_{\text {pred }}^{(i)}$ will be the best replacement time that balances the intervention costs incurred, and the reliability of the system in a renewal cycle.

\subsubsection{Policy performance assessment: Optimization of $T$}

To assess the performance of a maintenance policy, we have to rely on its optimal solution. Under the DR model, assessment of its performance is relative to the optimization of the inter-inspection time interval $T$ according to the cost criterion (6). The expected maintenance cost rate over an infinite time span of the DR policy can be computed by

$$
C_{D}^{\infty}(T)=\frac{E\left[C_{D}\left(S_{D}\right)\right]}{E\left[S_{D}\right]}=\frac{C_{p} P_{p, D}+C_{c}\left(1-P_{p, D}\right)+C_{i} E\left[N_{i, D}\right]+C_{i} E\left[W_{d, D}\right]}{E\left[S_{D}\right]},
$$

where $P_{p, D}, N_{i, D}$, and $W_{d, D}$ are respectively the preventive replacement probability, the number of inspections, and the system inactivity interval in a replacement cycle $S_{D}$ under the DR policy. $C_{D}^{\infty}(T)$ does not depend on the sequence of random measurements $\mathbf{Z}_{T_{0: i}}$ because the probability $P_{p, D}$, and the expected values of $N_{i, D}, W_{d, D}$, and $S_{D}$ are calculated based on the probability distribution of $\mathbf{Z}_{T_{0: i}}$.

Let's denote $T_{d}=\inf \left\{t \mid x_{t}>d\right\}$ as the random failure time of the system. Without loss of generality, we assume that the system is replaced at $T_{i+1}$, or at $T_{p r e d}^{(i)}$. Thus, the replacement cycle $S_{D}$ is expressed by

$$
S_{D}=\sum_{i=0}^{\infty} T_{i+1} \cdot 1_{\left\{T_{i}<T_{d}<T_{i+1}<T_{\text {pred }}^{(i)}\right\}}+\sum_{i=0}^{\infty} T_{\text {pred }}^{(i)} \cdot 1_{\left\{T_{i}<T_{\text {pred }}^{(i)}<T_{i+1}\right\}} .
$$

As such, its expected value is computed as

$$
E\left[S_{D}\right]=\sum_{i=0}^{\infty} T_{i+1} \cdot \operatorname{Pr}\left\{T_{i}<T_{d}<T_{i+1}<T_{\text {pred }}^{(i)}\right\}+\sum_{i=0}^{\infty} E\left[T_{\text {pred }}^{(i)} \cdot 1_{\left\{T_{i}<T_{\text {pred }}^{(i)}<T_{i+1}\right\}}\right]
$$


The probability of preventive replacement $P_{p, D}$ is given by

$$
P_{p, D}=\sum_{i=0}^{\infty} \operatorname{Pr}\left\{T_{i}<T_{\text {pred }}^{(i)}<T_{i+1}, T_{d}>T_{\text {pred }}^{(i)}\right\}
$$

From the last inspection time, the DR policy allows a system replacement (preventive or corrective) without inspection. So, the number of inspections $N_{i, D}$ in a renewal cycle is expressed by

$$
N_{i, D}=\sum_{i=0}^{\infty} i \cdot\left(1_{\left\{T_{i}<T_{d}<T_{i+1}<T_{\text {pred }}^{(i)}\right\}}+1_{\left\{T_{i}<T_{\text {pred }}^{(i)}<T_{i+1}\right\}}\right) .
$$

Hence, its expected value is given by

$$
E\left[N_{i, D}\right]=\sum_{i=0}^{\infty} i \cdot\left(\operatorname{Pr}\left\{T_{i}<T_{d}<T_{i+1}<T_{\text {pred }}^{(i)}\right\}+\operatorname{Pr}\left\{T_{i}<T_{\text {pred }}^{(i)}<T_{i+1}\right\}\right)
$$

And the length of the inactivity time interval in a replacement cycle is given as

$$
W_{d, D}=\sum_{i=0}^{\infty}\left(T_{\text {pred }}^{(i)}-T_{d}\right) \cdot 1_{\left\{T_{i}<T_{d}<T_{\text {pred }}^{(i)}<T_{i+1}\right\}}+\sum_{i=0}^{\infty}\left(T_{i+1}-T_{d}\right) \cdot 1_{\left\{T_{i}<T_{d}<T_{i+1}<T_{\text {pred }}^{(i)}\right\}} \cdot
$$

So its expected value is calculated as

$$
E\left[W_{d, D}\right]=\sum_{i=0}^{\infty} E\left[\left(T_{\text {pred }}^{(i)}-T_{d}\right) \cdot 1_{\left\{T_{i}<T_{d}<T_{\text {pred }}^{(i)}<T_{i+1}\right\}}\right]+\sum_{i=0}^{\infty} E\left[\left(T_{i+1}-T_{d}\right) \cdot 1_{\left\{T_{i}<T_{d}<T_{i+1}<T_{\text {pred }}^{(i)}\right\}}\right] .
$$

Therefore, the optimal problem of the DR policy is reduced to find the value of inter-inspection time interval $T_{\text {opt }}$ that minimizes the cost function $C_{D}^{\infty}(T)$ given by (11). That is,

$$
C_{D}^{\infty}\left(T_{\text {opt }}\right)=\min _{T}\left(C_{D}^{\infty}(T), T>0\right)
$$

To evaluate analytically the quantities $E\left[S_{D}\right], P_{p, D}, E\left[N_{i, D}\right]$, and $E\left[W_{d, D}\right]$, we need to know the mathematical formulas of the marginal distribution function of $s$-dependent variables $T_{d}$ and $T_{\text {pred }}^{(i)}$, as well as their joint distribution functions (see (12), (13), (14), and (15)). However, with the complexity of the considered model, the mathematical formulas of these distribution functions are intractable. Hence, one cannot derive completely the analytical cost model of the DR model, and a numerical approach has to be used to find the optimal solution of (16). The Monte Carlo simulation approach will be used for this purpose. 


\subsection{Quantile-based Inspection and Replacement model, QIR}

\subsubsection{Decision rule}

The DR policy is an effective policy to guarantee system availability, but its preventive replacements are still time-based decisions which can be less efficient than condition-based decisions [15]. Therefore, this section aims to develop a more sophisticated maintenance policy which can at the same time satisfy the condition-based aspect and the dynamic aspect. A natural solution is to simply give up the periodic inspection structure, and to apply a condition-based maintenance rule at the inspection times. The structure of such a maintenance decision rule in a renewal cycle can be described by the following scheme.

1. Inspect at time $T_{i}$.

2. If the system is failed, it is correctively replaced, and a new renewal cycle starts.

3. If it is still running, an estimation of degradation level at time $T_{i}, \hat{x}_{T_{i}}$, is reconstructed from the sequence of measurements $\mathbf{Z}_{T_{0: i}}$. If the estimated degradation level $\hat{x}_{T_{i}}$ overpasses a threshold $M$, the system is preventively replaced, and a new renewal cycle starts. Otherwise, nothing is done at $T_{i}$, and the decision is postponed until the next inspection planed at $T_{i+1}$.

4. Using the sequence of measurements $\mathbf{Z}_{T_{0: i}}$ given at current inspection $T_{i}$, the next inspection time $T_{i+1}$ is dynamically optimized in real time based on a reliability criterion [36] (Section 3.3.2). It guarantees that a given reliability level is preserved (the reliability has to be higher than a quantile $\alpha \in(0,1)$ over the time horizon $\left.\left[T_{i}, T_{i+1}\right]\right)$. As shown in [36], such an inspection scheme is a non-periodic inspection scheme, and it is called the quantile-based inspection.

The inspection times $T_{i+1}$ are recalculated at each inspection $T_{i}$ given the sequence of measurements $\mathbf{Z}_{T_{0: i}}$, so it can guarantee the dynamic property of a maintenance model. For this maintenance policy, the inspection time $T_{i+1}$, the preventive replacement degradation threshold $M$, and the quantile $\alpha$ are the three decision variables. Optimization of $M$ and $\alpha$ with the cost criterion $C^{\infty}$ given from (6) (see Section 3.3.3) provides a minimal expected maintenance cost rate to assess the performance of the policy. To enhance the parameter $\alpha$ that characterizes the dynamic inspection scheme, this decision rule is referred to in the following as QIR for quantile based inspection and replacement.

\subsubsection{Predictive replacement time estimation: real-time optimization of $T_{i}$}

For this inspection scheme, an inspection schedule is built dynamically using the predictive system reliability in a way similar to the work of Cui et al. in [36], and Yang et al. in [37]. These authors assume that the maintenance policy is used with perfect monitoring information, and availability 
criterion. Moreover, they do not mention the conditional aspect of the maintenance model. Therefore, we will extend the model to the case of imperfect measurement, real-time decisions, and taking into account the conditional aspect of the maintenance model. Given the sequence of measurements $\mathbf{Z}_{T_{0: i}}=\left\{z_{T_{0}}, z_{T_{1}}, \ldots, z_{T_{i}}\right\}, T_{0}=0$, at actual inspection $T_{i}$, the next inspection time $T_{i+1}$ is determined by, for $0<\alpha<1$,

$$
T_{i+1}=\sup _{j}\left\{T_{i}+j \Delta t: R\left(T_{i}+j \Delta t, \mathbf{Z}_{T_{0: i}}\right) \geq \alpha\right\}
$$

where $R\left(T_{i}+j \Delta t, \mathbf{Z}_{T_{0: i}}\right)$ is given from (9). Such an inspection time $T_{i+1}$ is a random variable that depends on the predetermined value of quantile $\alpha$, and the sequence of random measurements $\mathbf{Z}_{T_{0: i}}$.

\subsubsection{Policy performance assessment: Optimization of $M$ and $\alpha$}

Assessment of the performance of the QIR model is relative to optimization of preventive replacement threshold $M$ and the quantile $\alpha$ in the purpose of minimizing the global expected maintenance cost rate. Applying (6), the expected maintenance cost rate of the QIR policy is expressed by

$$
C_{Q}^{\infty}(\alpha, M)=\frac{E\left[C_{Q}\left(S_{Q}\right)\right]}{E\left[S_{Q}\right]}=\frac{C_{p} P_{p, Q}+C_{c}\left(1-P_{p, Q}\right)+C_{i} E\left[N_{i, Q}\right]+C_{i} E\left[W_{d, Q}\right]}{E\left[S_{Q}\right]}
$$

where $P_{p, Q}, N_{i, Q}$, and $W_{d, Q}$ are respectively the preventive replacement probability, the number of inspections, and the system inactivity interval in a replacement cycle $S_{Q}$ under the QIR policy. We will see that the probability $P_{p, Q}$, and the expected values of $N_{i, Q}, W_{d, Q}$, and $S_{Q}$ are calculated based on the probability distribution of $\mathbf{Z}_{T_{0: i}}$. Therefore, $C_{Q}^{\infty}(\alpha, M)$ does not depend on $\mathbf{Z}_{T_{0: i}}$.

Let's denote $T_{M}=\inf \left\{t \mid \widehat{x}_{t}>M\right\}$ as the random time to preventive replacement of the system. Under the QIR policy, a replacement is only possible at inspection times. So without loss of generality, if the system is replaced at $T_{i+1}$ given from (17), its replacement cycle can be expressed by

$$
S_{Q}=\sum_{i=0}^{\infty} T_{i+1} \cdot 1_{\left\{T_{i}<T_{M}<T_{i+1}\right\}}
$$

Therefore, the expected time to a system replacement is given by

$$
E\left[S_{Q}\right]=\sum_{i=0}^{\infty} E\left[T_{i+1} \cdot 1_{\left\{T_{i}<T_{M}<T_{i+1}\right\}}\right]
$$

The preventive replacement probability $P_{p, Q}$ is calculated by

$$
P_{p, Q}=\sum_{i=0}^{\infty} \operatorname{Pr}\left\{T_{i}<T_{M}<T_{i+1}<T_{d}\right\}
$$


Because the system failure is assumed to be self-announcing, no additional inspection is needed before a corrective replacement, counting from the last inspection. However, as the preventive replacement is decided based on the estimation of the hidden degradation level, it always requires inspecting the system condition before a replacement. Thus the number of inspections $N_{i, Q}$ effected in a renewal cycle is expressed by

$$
N_{i, Q}=\sum_{i=0}^{\infty}(i+1) \cdot 1_{\left\{T_{i}<T_{M}<T_{i+1}<T_{d}\right\}}+i \cdot 1_{\left\{T_{i}<T_{M}<T_{d}<T_{i+1}\right\}}
$$

Hence, the expected value is given by

$$
E\left[N_{i, Q}\right]=\sum_{i=0}^{\infty}(i+1) \cdot \operatorname{Pr}\left\{T_{i}<T_{M}<T_{i+1}<T_{d}\right\}+\sum_{i=0}^{\infty} i \cdot \operatorname{Pr}\left\{T_{i}<T_{M}<T_{d}<T_{i+1}\right\}
$$

And the length of inactivity time interval in a replacement cycle can be expressed as

$$
W_{d, Q}=\sum_{i=0}^{\infty}\left(T_{i+1}-T_{d}\right) \cdot 1_{\left\{T_{i}<T_{M}<T_{d}<T_{i+1}\right\}}
$$

Therefore, the expected value is given by

$$
E\left[W_{d, Q}\right]=\sum_{i=0}^{\infty} E\left[\left(T_{i+1}-T_{d}\right) \cdot 1_{\left\{T_{i}<T_{M}<T_{d}<T_{i+1}\right\}}\right]
$$

Consequently, the optimal problem of the QIR policy is reduced to find the optimal values $M_{\text {opt }}$ and $\alpha_{\text {opt }}$ that minimize the cost function given by (18); that is,

$$
C_{Q}^{\infty}\left(\alpha_{o p t}, M_{o p t}\right)=\min _{\alpha, M}\left(C_{Q}^{\infty}(\alpha, M): 0<\alpha<1,0<M<d\right)
$$

We can see that it is intractable to evaluate analytically the $E\left[S_{Q}\right], P_{p, Q}, E\left[N_{i, Q}\right]$, and $E\left[W_{d, Q}\right]$ because the mathematical functions of the marginal distribution and the joint distribution of $T_{i+1}, T_{M}$, and $T_{d}$ (see (19), (20), (21), and (22)) are not trivial to obtain. Thus, the analytical cost model of the QIR model is not completely derived. In the following, a Monte Carlo simulation will be used to find the optimal solution of (23).

\subsection{Benchmark maintenance models}

To assess the performance of the new policies, the two more classical maintenance policies (i.e., BR policy and PIR policy) considered by Huynh et al. in [15] are used as benchmarks. 


\subsubsection{Block replacement model, BR}

The BR policy represents a maintenance policy without inspection. It is a special case of the class of classical TBM policies whose decision rule is based only on the calendar time, or the age of the system, and on the initial knowledge of the statistical information on its lifetime. Under the BR policy, the system is replaced at regular time intervals $T$, either preventively if it is still running at the end of the replacement interval, or correctively if a failure occurred since the last replacement. The replacement period $T$ is the only decision variable for this policy. Because the system is periodically replaced regardless of the system condition, the BR policy is referred to as a non-condition-based, non-dynamic policy.

\subsubsection{Periodic inspection and replacement model, PIR}

Compared to the BR policy, the PIR policy is more sophisticated in the sense that the maintenance decision is based on the condition monitoring information returned by inspections on the system. For this policy, the system is periodically inspected with period $T$. At each inspection $T_{i}=i T$, if the crack depth $\hat{x}_{T_{i}}$ as estimated from the sequence of measurements $\mathbf{Z}_{T_{0: i}}$ exceeds a threshold $M$, then a preventive replacement is performed; but if the system has already failed, then it is correctively replaced. The inter-inspection interval $T$ and the preventive threshold $M$ are the decision variables of this policy. Although the maintenance decision of the PIR policy is based on system conditions, and its replacement times do not adapt to the system state, it is a maintenance policy with condition-based, non-dynamic aspects.

\subsection{Synthesis of maintenance rules characteristics}

The summarized characteristics of the considered maintenance models are listed in Table I.

We can see that the fundamental difference of DR and QIR decision rules compared to the PIR and $\mathrm{BR}$ ones is that the first group allows for dynamic preventive replacement with real-time optimization.

\section{Numerical solution for maintenance models}

The theoretical developments in Section 3 show that evaluating the maintenance cost models requires some density functions built from the sequence of noisy measurements. Because analytical formulas of these quantities are not trivial (often impossible), we propose in this section numerical methods to overcome this difficulty. The PF approach is used for the real-time optimization, while a Monte Carlo simulations approach is used to assess the performance of maintenance models. 
Table I: Summary of the considered maintenance policies

\begin{tabular}{|c|c|c|c|c|c|}
\hline \multirow{2}{*}{ Policy } & \multirow{2}{*}{\multicolumn{2}{|c|}{$\begin{array}{c}\text { Type of preventive } \\
\text { replacement }\end{array}$}} & \multicolumn{2}{|c|}{ Replacement time } & \multirow{2}{*}{ Inspection time } \\
\hline & & & Preventive & Corrective & \\
\hline$\overline{\mathrm{DR}}$ & & Dynamic & It depends ${ }^{a}$ & Non-dynamic \\
\hline QIR & \multicolumn{2}{|c|}{ Condition-based } & Dynamic & Dynamic & Dynamic \\
\hline $\mathrm{BR}$ & \multicolumn{2}{|c|}{ Time-based } & Non-dynamic & Non-dynamic & $\varnothing$ \\
\hline PIR & \multicolumn{2}{|c|}{ Condition-based } & Non-dynamic & Non-dynamic & Non-dynamic \\
\hline \multirow{2}{*}{ Policy } & \multicolumn{3}{|c|}{ Real-time optimization } & \multicolumn{2}{|c|}{ Optimization } \\
\hline & Parameters & & iterions & Parameters & Criterions \\
\hline $\mathrm{DR}$ & $T_{\text {pred }}^{(i)}$ & & st $C_{\text {pred }}$ & $T$ & Cost $C^{\infty}$ \\
\hline QIR & $T_{i+1}$ & Reliability & over $\left(T_{i}, T_{i+1}\right)$ & $\alpha, M$ & Cost $C^{\infty}$ \\
\hline $\mathrm{BR}$ & $\varnothing$ & & $\varnothing$ & $T$ & Cost $C^{\infty}$ \\
\hline PIR & $\varnothing$ & & $\varnothing$ & $T, M$ & Cost $C^{\infty}$ \\
\hline
\end{tabular}

\footnotetext{
${ }^{a}$ Corrective replacement is effected sometimes at inspection time $T_{i}$, sometimes at predictive replacement time $T_{\text {pred }}^{(i)}$.
}

\subsection{Particle filtering state estimation and prognostic}

Stochastic filtering [38], proportional hazards modeling [39], and hidden Markov models [40] are the three common data-driven approaches for inferring the underlying system state from noisy monitoring data [11]. The proportional hazards model is restricted to its proportional assumption, and the hidden Markov model is applied only for discrete state variables. The stochastic filtering approach can overcome these drawbacks. Moreover, because the stochastic filtering uses the full history of condition monitoring data, it outperforms the others [11]. The performance of stochastic filtering is limited with high dimension data. However, this limit is not the problem in our case study because the degradation phenomenon is a single fatigue crack growth path.

$\mathrm{PF}$ is a numerical version of stochastic filtering. It is a Monte Carlo-based computation tool particularly useful for optimal estimation and prediction problems in non-linear, non-Gaussian processes [11]. In comparison with standard approximation methods, such as the popular Extended Kalman Filter, the principal advantage of particle methods is that they do not rely on any local linearization technique or any crude functional approximation [41]. The price that must be paid for this flexibility is computationally expensive. However, thanks to the availability of ever-increasing computational power, these methods are already used in real-time applications appearing in fields as diverse as chemical engineering, computer vision, financial econometrics, target tracking, and robotics [42]. Here, we use a quite simple PF approach to estimate in real-time the crack depth of the system, and predict the conditional reliability $R\left(T_{i}+l \Delta t, \mathbf{Z}_{T_{0: i}}\right)$. 


\subsubsection{Particle filtering state estimation}

The robustness of PF leads us to share the similar simulation approach of Zio et al. in [18], [22] to estimate in real-time the system state at discrete inspection times. Because the paper does not focus on computation methods, we do not explain the principle of the considered PF. Interested readers can refer to [18], [22] for the detailed development, or to [43], [44] for other PF estimation approaches. Instead, we present explicitly the PF algorithm that is not introduced in [18], [22].

The algorithm uses the prior distribution derived from (2) as the importance function, and the deterministic re-sampling method [45] to limit the inherent degeneracy problem in the PF approach. As such, the real-time state estimation procedure, given the sequence of measurements $\mathbf{Z}_{T_{0: i}}=\left\{z_{T_{0}}, z_{T_{1}}, \ldots, z_{T_{i}}\right\}$, can be resumed by Algorithm 1.

Algorithm 1 Generic particle filter (SIS/Re-sampling Monte Carlo filter) for system state estimation

1. Initialization: $\forall k=1, \ldots, N_{s}$

Generate $\widetilde{x}_{t_{0}}^{(k)} \sim f\left(x_{t_{0}}\right)$, and set $w_{t_{0}}^{(k)}=1 / N_{s}$.

2. Given $\left\{\widehat{x}_{t_{i-1}}^{(k)}, 1 / N_{s}\right\}_{k=1}^{N_{s}}$, at $t_{i}>0$, do the following.

(a) Prediction step: Generate $N_{s}$ particles $\widetilde{x}_{t_{i}}^{(k)}$ from (2).

(b) If $t_{i} \neq T_{i}$ (not at inspection), set $\left\{\widehat{x}_{t_{i}}^{(k)}, w_{t_{i}}^{(k)}\right\}_{k=1}^{N_{s}}=\left\{\widetilde{x}_{t_{i}}^{(k)}, 1 / N_{s}\right\}_{k=1}^{N_{s}}$

(c) If $t_{i}=T_{i}$ (at inspection), do the following.

i. Update step: $w_{t_{i}}^{(k)} \propto f\left(z_{T_{i}} \mid \widetilde{x}_{t_{i}}^{(k)}\right)$.

ii. Re-sampling step: Deterministic re-sampling $\left\{\widetilde{x}_{t_{i}}^{(k)}, w_{t_{i}}^{(k)}\right\}_{k=1}^{N_{s}}$ to obtain $\left\{\widehat{x}_{t_{i}}^{(k)}, 1 / N_{s}\right\}_{k=1}^{N_{s}}$.

iii. Set $\left\{\widehat{x}_{T_{i}}^{(k)}, w_{T_{i}}^{(k)}\right\}_{k=1}^{N_{s}}=\left\{\widehat{x}_{t_{i}}^{(k)}, 1 / N_{s}\right\}_{k=1}^{N_{s}}$.

3. Estimation at inspection $T_{i}$ :

(a) Estimated pdf: $\widehat{f}\left(x_{T_{i}} \mid \mathbf{Z}_{T_{0: i}}\right)=\left(\sum_{k=1}^{N_{s}} \delta_{\widehat{x}_{T_{i}}^{(k)}}\left(x_{T_{i}}\right)\right) / N_{s}$.

(b) Estimated state:

$$
\widehat{x}_{T_{i}}=\int x_{T_{i}} f\left(x_{T_{i}} \mid \mathbf{Z}_{T_{0: i}}\right) d x_{T_{i}} \simeq\left(\sum_{k=1}^{N_{s}} \int x_{T_{i}} \delta_{\widehat{x}_{T_{i}}}\left(x_{T_{i}}\right) d x_{T_{i}}\right) / N_{s}=\left(\sum_{k=1}^{N_{s}} \widehat{x}_{T_{i}}^{(k)}\right) / N_{s} .
$$

To illustrate the robustness of the proposed PF algorithm, we consider two deviation levels in the measured data. One is low compared to the variance of the degradation process itself (i.e. $\sigma_{v}=0.5$ ), and one is high $\left(\sigma_{v}=1.5\right)$. The estimated states $\widehat{x}_{T_{i}}$ at periodic inspections times $T_{i}$ are compared in both cases to the true states $x_{T_{i}}$, and to the measurements $z_{T_{i}}$. The numerical results presented in Fig. 1 correspond to $C=0.015, n=0.35, \beta=3.9, \sigma_{\omega}^{2}=2.53, \Delta t=1, \beta_{0}=0.06$, and $\beta_{1}=1.25$. The critical material thickness is $d=6$, samples number is $N_{s}=3000$, and inter-inspection time interval is $T=10$. Because the degradation is estimated by its expected value conditional on the sequence of noisy measurements, moreover the PF algorithm is an approximation of the optimal Bayesian estimate, 
the error between estimated state and the true degradation state is unavoidable. As depicted in Fig. 1,

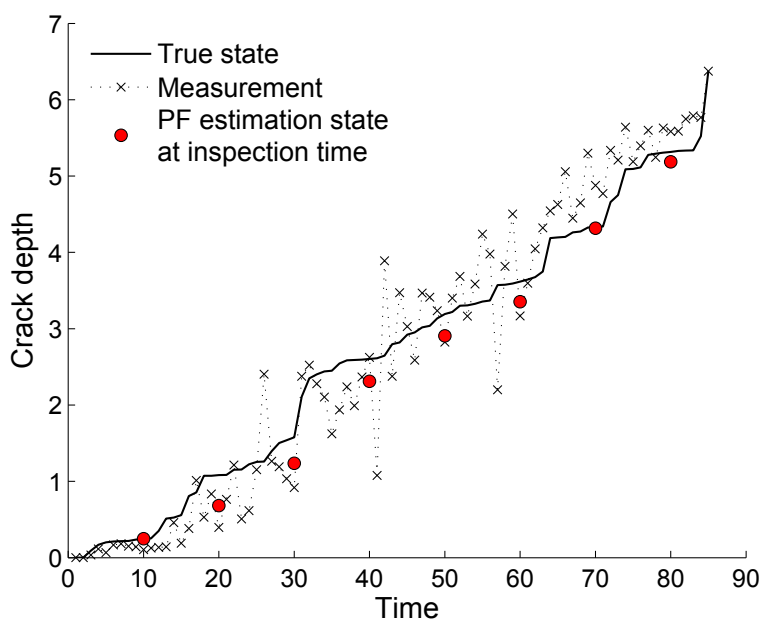

(a) Standard deviation: $\sigma_{v}=0.5$.

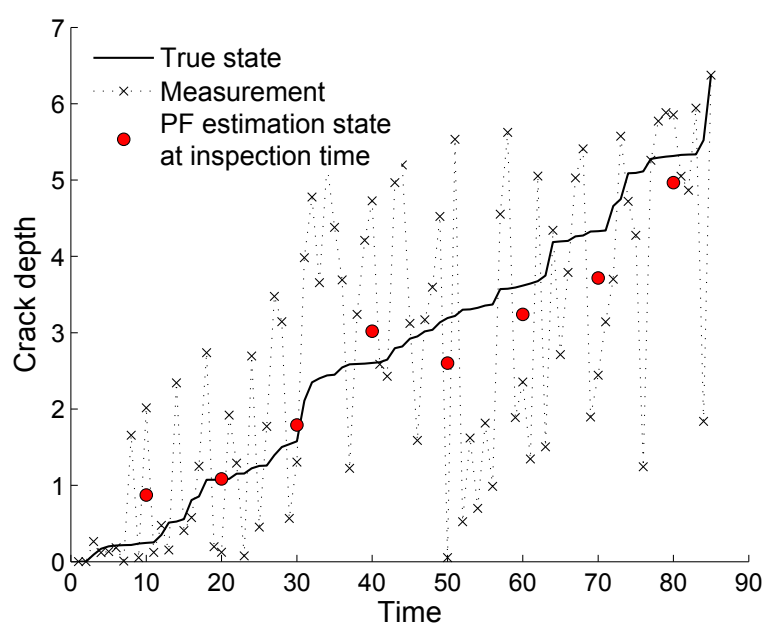

(b) Standard deviation: $\sigma_{v}=1.5$.

Figure 1: State estimation by generic particle filter algorithm.

this error increases with respect to the standard deviation of the measurement. However, the estimated state can still approximate the true degradation path more closely compared to the measured data.

\subsubsection{Particle filtering prognostic}

This section aims to provide a numerical solution of the conditional reliability $R\left(T_{i}+j \Delta t, \mathbf{Z}_{T_{0: i}}\right)$ that is a key element in the real-time optimization procedure of the DR policy and the QIR policy. If the measurement is perfect (i.e. $\mathbf{Z}_{T_{0: i}}$ reflects exactly the true degradation state of system), the analytical formulas of $R\left(T_{i}+j \Delta t, \mathbf{Z}_{T_{0: i}}\right)$ can be derived because the probability law of the degradation increment is known (3). In the present paper, the measurement $\mathbf{Z}_{T_{0: i}}$ is imperfect. Hence, the analytical formulation is intractable. However, given $\mathbf{Z}_{T_{0: i}}$, the system states can be reconstructed through Algorithm 1. Moreover, because the future measurements have obviously not yet been collected, the only thing related to the future condition of the system that is known is the dynamic model of state evolution (i.e. (2)). Therefore, we use the estimated state at the current inspection time $T_{i}$ (i.e. $\widehat{x}_{T_{i}}$ or the set of $N_{s}$ particles $\left.\widehat{x}_{T_{i}}^{(k)}\right)$, and (2) to provide an empirical approximation of $R\left(T_{i}+j \Delta t, \mathbf{Z}_{T_{0: i}}\right)$.

The first stage regards the recursive generation of sets of $N_{s}$ particles up to the time of interest (e.g. $\quad \widetilde{x}_{T_{i}+j \Delta t}^{(k)}$ at time $\left.T_{i}+j \Delta t\right)$ from $N_{s}$ particles $\widehat{x}_{T_{i}}^{(k)}$ using (2). Then, the conditional reliability $R\left(T_{i}+j \Delta t, \mathbf{Z}_{T_{0: i}}\right)$ given from (9) can be approximated by

$$
R\left(T_{i}+j \Delta t, \mathbf{Z}_{T_{0: i}}\right)=\frac{\operatorname{Pr}\left\{x_{T_{i}+j \Delta t}<d \mid \mathbf{Z}_{T_{0: i}}\right\}}{\operatorname{Pr}\left\{x_{T_{i}}<d \mid \mathbf{Z}_{T_{0: i}}\right\}} \simeq \frac{\left.\sum_{m=1}^{N_{s}} 1_{\left\{\widetilde{x}_{T_{i}+j \Delta t}^{(m)}<d\right.}\right\}}{\sum_{n=1}^{N_{s}} 1_{\left\{\widehat{x}_{T_{i}}^{(n)}<d\right\}}}
$$

The numerator (resp. denominator) of (24) represents the number of sampled particles which are still 
running at time $T_{i}+j \Delta t$ (resp. $\left.T_{i}\right)$. Knowing this approximation of $R\left(T_{i}+j \Delta t, \mathbf{Z}_{T_{0: i}}\right)$, we can determine in real time the predictive replacement time $T_{p r e d}^{(i)}$, and the inspection time $T_{i+1}$, at each inspection $T_{i}$, according to (10), and (17) respectively.

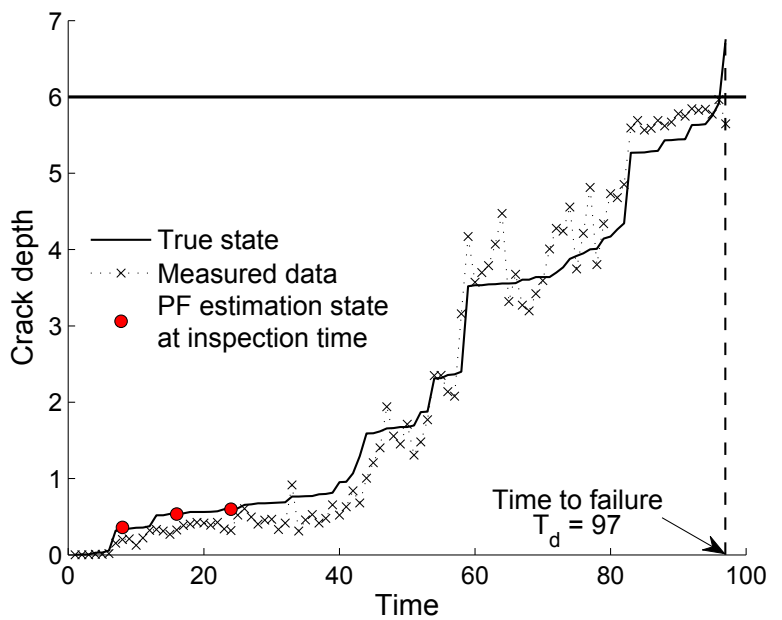

(a) Simulated crack depth evolution.

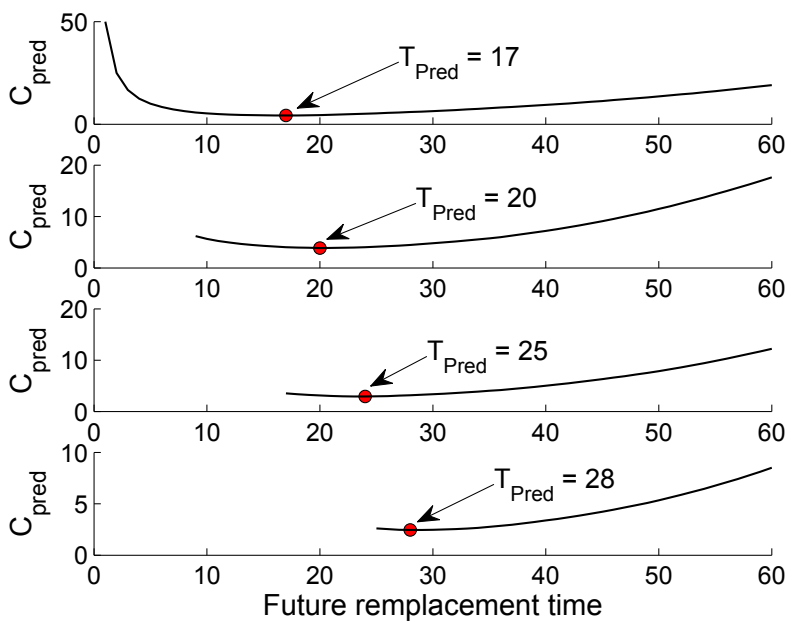

(b) Cost curves to predict suitable replacement time.

Figure 2: Predictive replacement times in DR policy $-T=8, C_{c}=100, C_{p}=50, C_{d}=250, C_{i}=3$.

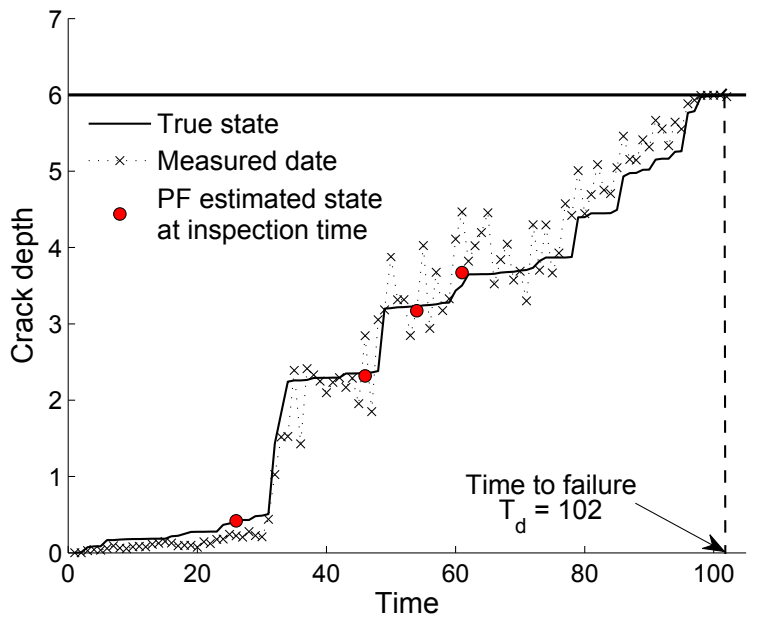

(a) Simulated crack depth evolution.

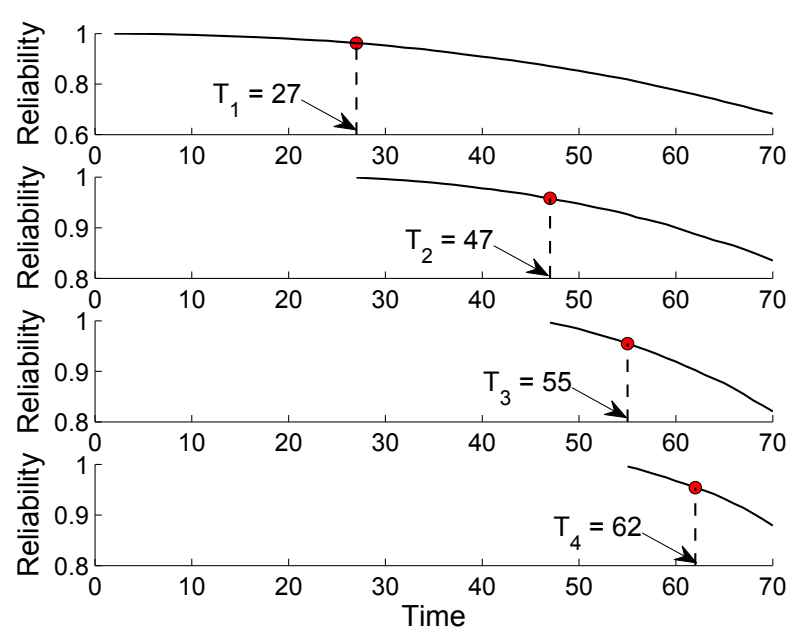

(b) Reliability curves to predict inspection time.

Figure 3: Predictive inspection times in QIR policy - $\alpha=0.96$.

The illustrations of real-time optimization of $T_{\text {pred }}^{(i)}$ and $T_{i+1}$ are based on the set of parameters $C=0.0047, n=0.35, \beta=3.9, \sigma_{\omega}^{2}=4.8326, \Delta t=1, \beta_{0}=0.06, \beta_{1}=1.25, \sigma_{v}^{2}=0.0625$, the critical material thickness $d=6$, and $N_{s}=3000$. Figs. $2 \mathrm{~b}$, and 3b depict respectively the cost curves, and the curves of conditional reliability to predict the replacement times $T_{p r e d}^{(i)}$, and the inspection times $T_{i+1}$ at different inspection times corresponding to the single path of crack depth evolution in Figs. 2a, and 3a. The time for each optimisation of $T_{p r e d}^{(i)}$ and $T_{i+1}$ is negligible (on the order of seconds). 


\subsection{Monte Carlo simulation for maintenance optimization}

We have shown in Section 3 that analytical cost models of the DR policy and the QIR policy are not trivial to obtain due to the complexity of the considered degradation and measurement model. We must therefore resort to stochastic Monte Carlo simulation to approximate their optimal solutions. Thus, the long run expected maintenance cost rate given from (6) is rewritten to

$$
C^{\infty}=\frac{E[C(S)]}{E[S]}=\lim _{N \rightarrow \infty} \frac{\frac{1}{N} \sum_{n=1}^{N} C\left(S^{(n)}\right)}{\frac{1}{N} \sum_{n=1}^{N} S^{(n)}} \simeq \frac{\sum_{n=1}^{N_{h}} C\left(S^{(n)}\right)}{\sum_{n=1}^{N_{h}} S^{(n)}}, \text { with } N_{h} \text { high enough }
$$

where $N_{h}$ is the number of simulation histories, and $S^{(n)}$ is the length of the first renewal cycle of the $n$-th history. Algorithm 2 describes the simulation procedure that represents how to obtain the long run expected maintenance cost rate $C^{\infty}$ according to (25).

Algorithm 2 Simulation algorithm to determine $C^{\infty}$ according to (25)

1. Initialization: $S=0, C=0$.

2. Step 1: Generate a history (e.g. the $n$-th history) of degradation and measurements according to (2), and (4).

3. Step 2: Determine the length of the first renewal cycle $S^{(n)}$ of the $n$-th history and the corresponding maintenance cost incurred $C\left(S^{(n)}\right)$ (see respectively Algorithms 3 and 4 for detail simulation procedures of DR policy and QIR policy).

4. Step 3: Calculate $C=C+C\left(S^{(n)}\right), S=S+S^{(n)}, C_{\infty}=C / S$, and decide

(a) if $C_{\infty}$ converge, end of simulation algorithm;

(b) otherwise, turn back to Step 1.

5. Output: Converged value of $C_{\infty}$.

In the remainder of this section, we use a degradation process with a quite linear mean path for numerical illustrations. Note that a degradation process given from the Paris-Erdogan model (1) always has a convex mean path. The choice of a quasilinear mean degradation process path, as in this paper, is justified by the important number of applications corresponding to this kind of trend [2], and for the sake of clarity in the discussions. Obviously, the same kind of results can be obtained in the non-linear case, with the same kind of discussions. Hence, the same data set in the example of Section 4.1.2 is reused here by adding the number of simulated histories $N_{h}=4000$ (the value of $C_{\infty}$ is converged when $\left.N_{h}=4000\right)$, and the intervention costs $C_{p}=50, C_{c}=100$, and $C_{d}=250$.

\subsubsection{Numerical solution for the DR model}

This section shows how to obtain the length of the renewal cycle $S_{D}^{(n)}$, and the maintenance cost $C\left(S_{D}^{(n)}\right)$ (i.e., step 2 of Algorithm 2), by applying the decision rule of the DR policy on the $n$-th history. The simulation procedure is described by Algorithm 3. 
Algorithm 3 Simulation algorithm to determine $S_{D}^{(n)}$, and $C\left(S_{D}^{(n)}\right)$, according to the DR policy

1. Initialization: $T, T_{i}=0, \mathbf{Z}_{T_{0: i}}=0$.

2. Step 1: Decide at time $T_{i}$

(a) If the system fails, do corrective replacement: $C\left(S_{D}^{(n)}\right)=(i-1) C_{i}+C_{c}+C_{d}\left(T_{i}-T_{d}^{(n)}\right)$ where $T_{d}^{(n)}$ is the failure time of the $n$-th history, $S_{D}^{(n)}=T_{i}$, and end of simulation algorithm.

(b) Otherwise, go to Step 2.

3. Step 2: Inspect the system to obtain $\mathbf{Z}_{T_{0: i}}$, predict the predictive replacement time $T_{\text {pred }}^{(i)}$ using equations (7), (8), (24) and Algorithm 1, and decide

(a) if $T_{\text {pred }}^{(i)}>T_{i}+T$, set $T_{i}=T_{i}+T$, and turn back to Step 1;

(b) otherwise, go to Step 3.

4. Step 3: Decide at time $T_{\text {pred }}^{(i)}$

(a) if the system fails, do corrective replacement: $C\left(S_{D}^{(n)}\right)=(i-1) C_{i}+C_{c}+C_{d}\left(T_{\text {pred }}^{(i)}-T_{d}^{(n)}\right)$, $S_{D}^{(n)}=T_{p r e d}^{(i)}$, and end of simulation algorithm.

(b) Otherwise, do preventive replacement: $C\left(S_{D}^{(n)}\right)=(i-1) C_{i}+C_{p}, S_{D}^{(n)}=T_{p r e d}^{(i)}$, and end of simulation algorithm.

5. Output: Values of $S_{D}^{(n)}$, and $C\left(S_{D}^{(n)}\right)$.

Applying Algorithms 2 and 3 to the selected data set, the expected maintenance cost curves of the DR policy corresponding to different values of the inspection cost are depicted as in Fig. 4. The time

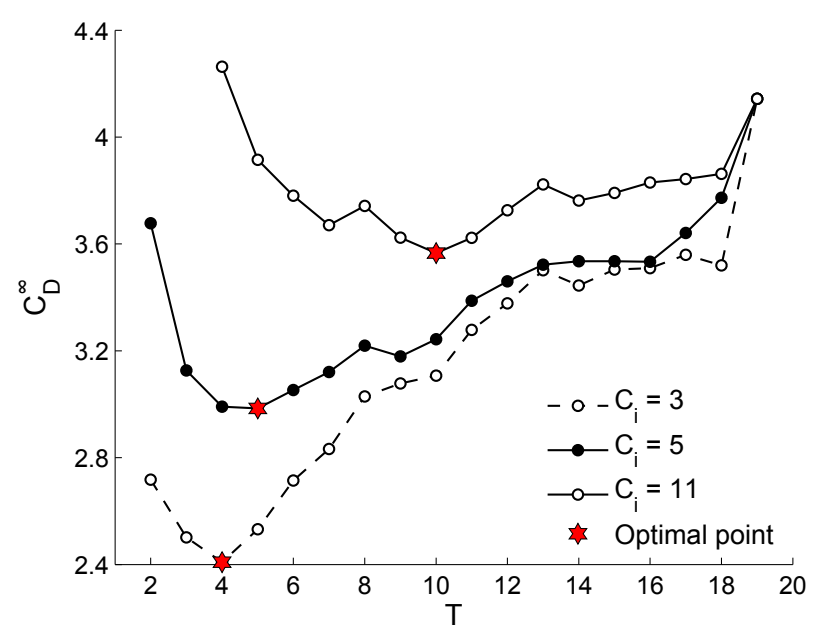

Figure 4: Expected maintenance cost of DR policy versus inter-inspection interval.

for optimization is around 24 hours. The figure shows clearly that one can obtain an optimal solution under this maintenance policy. All maintenance cost curves converge to a value when an inter-inspection interval $T$ becomes large enough. This result can be explained by the fact that the maintenance policy becomes almost identical to a BR policy (see Subsection 3.4.1) when the inter-inspection interval is large enough; the first inspection $T_{1}$ is performed at time $T$, and because $T$ is high, the system fails with a high probability. It is replaced by a new one, and the next inspection is performed at $T_{2}=2 T$, so the 
same situation is observed; because $T$ is high, the system fails, and so on. Thus, the converged value is just the optimal value of the BR policy, which has the same configuration.

\subsubsection{Numerical solution for the QIR model}

This section shows how to obtain the length of the renewal cycle $S_{Q}^{(n)}$, and the maintenance cost $C\left(S_{Q}^{(n)}\right)$ (i.e. step 2 of Algorithm 2) by applying the decision rule of the QIR policy on the $n$-th history. The simulation procedure is described by Algorithm 4.

Algorithm 4 Simulation algorithm to determine $S_{Q}^{(n)}$, and $C\left(S_{Q}^{(n)}\right)$ according to the QIR policy

1. Initialization: $\alpha, M, T_{i}=0, \mathbf{Z}_{T_{0: i}}=0$.

2. Step 1: Decide at time $T_{i}$ the following.

(a) If the system fails, do corrective replacement: $C\left(S_{Q}^{(n)}\right)=(i-1) C_{i}+C_{c}+C_{d}\left(T_{i}-T_{d}^{(n)}\right)$ where $T_{d}^{(n)}$ is the failure time of the $n$-th history, $S_{Q}^{(n)}=T_{i}$, and end of simulation algorithm. (b) Otherwise, go to Step 2.

3. Step 2: Inspect the system to obtain $\mathbf{Z}_{T_{0: i}}$, estimate the system degradation level $\widehat{x}_{T_{i}}$ according to algorithm 1 , and decide the following.

(a) If $\widehat{x}_{T_{i}}>M$, do preventive replacement: $C\left(S_{Q}^{(n)}\right)=i C_{i}+C_{p}, S_{Q}^{(n)}=T_{i}$, and end of simulation algorithm.

(b) Otherwise, predict the next inspection time using (17), (24), and Algorithm 1; and turn back to Step 1.

4. Output: Values of $S_{Q}^{(n)}$, and $C\left(S_{Q}^{(n)}\right)$.

Applying Algorithms 2 and 4 to the selected data set, Fig. 5 shows a numerical example of the optimization results when $C_{i}=5$. Fig. 5 a sketches the shape of the expected maintenance cost rate of the QIR policy when the preventive replacement threshold $M$ and the quantile $\alpha$ vary. Fig. 5b shows that one obtains the optimal value $C_{Q \text { opt }}^{\infty}=2.8008$ at $\alpha_{\text {opt }}=0.9915$, and $M_{\text {opt }}=2.5$.

The examples above show the optimal solutions of the maintenance policies for a special case. To have a thorough analysis on the effectiveness of these maintenance policies, as well as on the benefit of applying the online prognostic information in maintenance decisions, the next two sections will make comparisons of the expected maintenance cost among the considered maintenance models under different possible configurations. As stated in the introduction, the aim is to investigate at the same time and for the same case study the relevance of different maintenance rules, taking into account the monitoring quality, the maintenance and inspection costs, and the characteristics of the degradation processes.

\section{Impact of measured information uncertainty on maintenance decision-making}

As mentioned in Section 4.1.1, the higher the standard deviation $\sigma_{v}$ of the measured data, the more the imprecision of the state estimation increases (see Fig. 1). Because the decision rules of the considered 


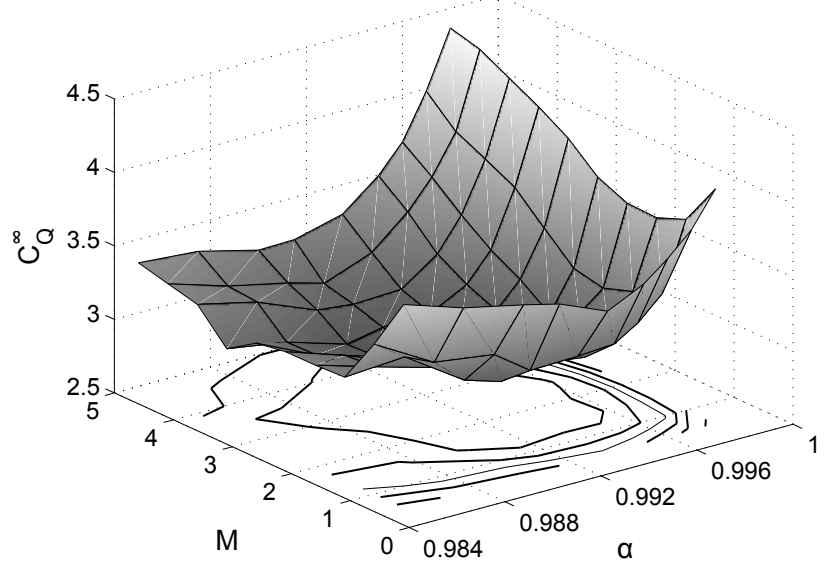

(a) Mesh of expected maintenance cost.

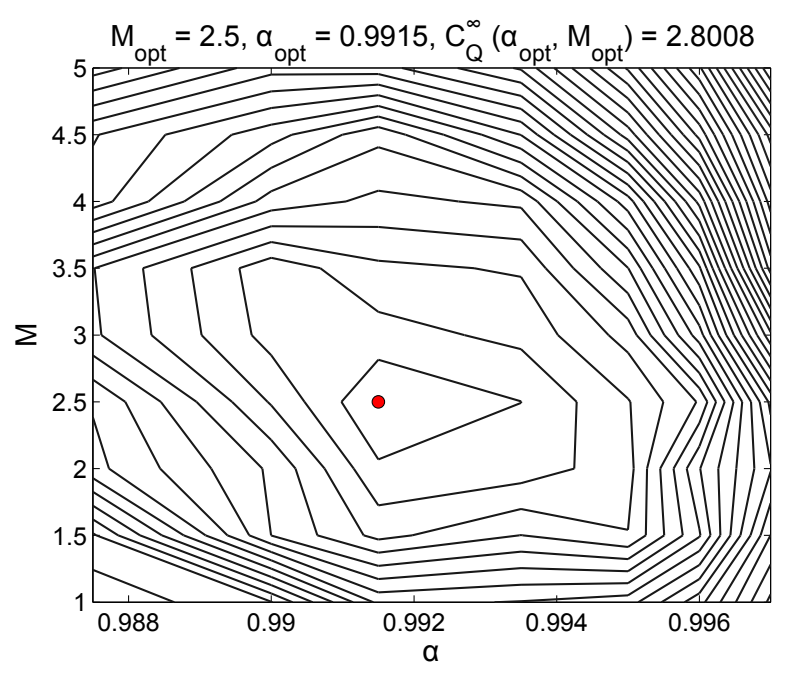

(b) Iso-level of expected maintenance cost.

Figure 5: Expected maintenance cost curves of the QIR policy.

maintenance models implement based on the estimated states, the errors in measurement may reduce the effectiveness of maintenance policies. Therefore, the focus of this section is the impact of the monitoring quality. The natural questions that arise are numerous.

- Does a maintenance policy based on information of conditions still retain its advantage compared to a TBM policy?

- Is a maintenance decision based on the reconstructed degradation state $\hat{x}_{T_{i}}$ more effective than a decision directly based on the measured data $z_{T_{i}}$ ?

○ How does one limit the negative impact of uncertainty on the maintenance decision?

For our case study, these questions are closely linked to the quality of the ultrasonic measure technique, and to the PF estimation step. To quantify the impact of measured information uncertainty on the maintenance decision, we perform a comparison on the optimal expected maintenance cost among the maintenance policies (BR, PIR, DR, and QIR) as a function of the standard deviation $\sigma_{v}$. To show the relevance of a model taking into account the monitoring characteristics, we also consider the three following situations for the maintenance decision rules:

1. The decision for the preventive replacement at time $T_{i}$ is based on the true system state $x_{T_{i}}$. This situation is unrealistic, but is considered as a reference. It is referred in the following as the true state-based policy case.

2. The decision for the preventive replacement at time $T_{i}$ is based on the estimated state reconstructed from measured data $\hat{x}_{T_{i}}$. It corresponds to a normal use of our model. It is referred in the following as the estimated state-based policy case. 
3. The decision for the preventive replacement at time $T_{i}$ relies directly on the measured data $z_{T_{i}}$. This case applies when the measured data are considered to be perfect, and the monitoring characteristics are neglected. It is referred as the measured state-based policy case.

The BR policy which does not depend on the inspection is used as a benchmark policy to investigate the influence of noisy condition monitoring information, and to indicate which type of maintenance policy should be applied according to the uncertainty of measured data.

\subsection{Numerical results}

Because the study focuses only on the impact of the monitoring quality on the performance of maintenance policies, we fix the system characteristics and the maintenance costs with the data set $C=0.015, n=0.35, \beta=3.9, \sigma_{\omega}^{2}=2.53, \Delta t=1, \beta_{0}=0.06, \beta_{1}=1.25, d=6, C_{i}=5, C_{p}=50$, $C_{c}=100$, and $C_{d}=250$. The sample number, and simulated histories number are $N_{s}=3000$, and $N_{h}=4000$ respectively. Then we vary the value of the standard deviation $\sigma_{v}$ from 0 to 3.5 with unit step 0.5 , and observe the evolution of the optimal expected maintenance cost rate. The numerical results are shown in fig. 6 .

\subsection{Analysis and discussion of the numerical results}

The results show clearly that, for whatever estimated or measured state-based policies, their optimal maintenance costs increase with respect to the increasing error in measurements. The three policies with maintenance decisions based on the measured state can lead to substantial maintenance cost savings compared to the BR policy, for a reasonable deviation in measured data. This benefit becomes no longer true when the uncertainty in measurement becomes very high (remember that $\sigma_{v}>3.5$ is a very high variance compared to the process variance if we look at Fig. 1), because in this case the measurement can no longer represent the true evolution of the degradation process. This inaccurate information can cause unadapted maintenance decisions, so a BR maintenance policy that ignores the online information will be more efficient. One can note that this phenomenon is more clearly visible in the PIR policy than in the DR and QIR policies. Hence, dynamic maintenance policies are more robust than periodic ones to measured noise. In other words, the dynamic aspect of the maintenance structure can limit the negative impact of measurement uncertainty. Another way to reduce this impact is to make maintenance decisions based on the estimated state reconstructed from the measured data. The Fig. 6 shows that, for very high (and unrealistic) measurement noise, the three policies based on the estimated state $\hat{x}_{T_{i}}$ are still more efficient than if they were based on the observed state $z_{T_{i}}$, and they have not yet exceeded the BR cost. 


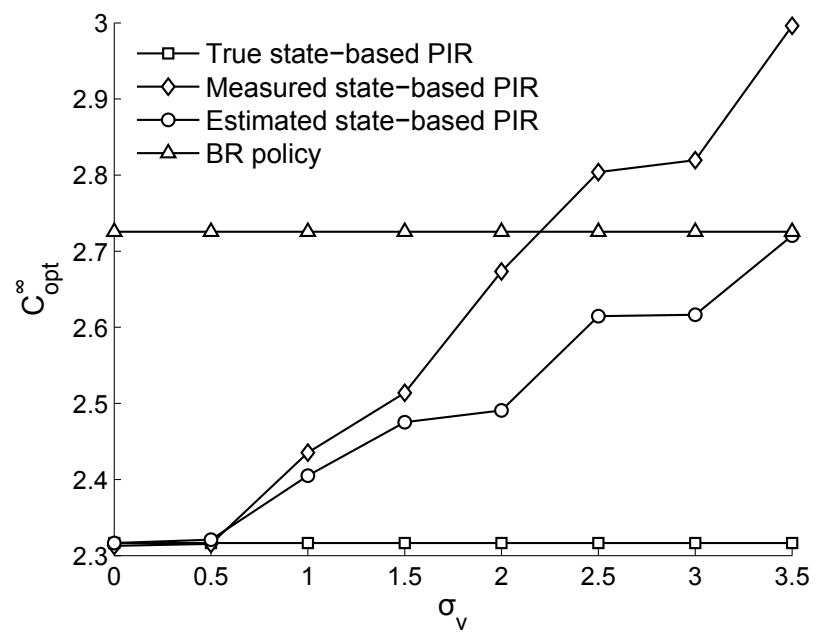

(a) PIR policy v.s. BR policy.

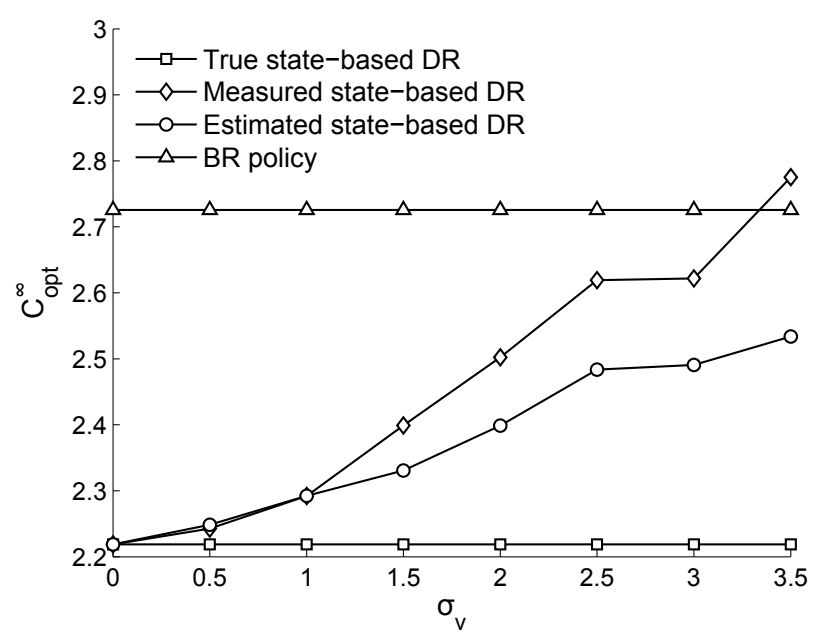

(b) DR policy v.s. BR policy.

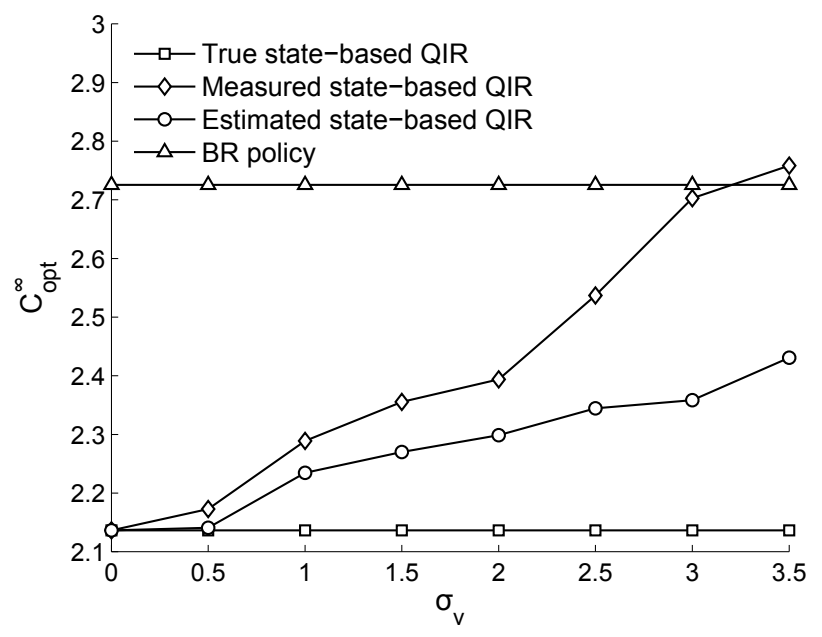

(c) QIR policy v.s. BR policy.

Figure 6: Impact of the measured data uncertainty on maintenance decision-making.

This study completes the conclusions in [23], [15] on the performance, and on the choice between CBM and TBM in the case of imperfect condition monitoring; the CBM policy is still more profitable than the TBM policy for a reasonable level of condition monitoring errors. A dynamic maintenance structure, and a decision based on the estimated degradation constructed from the measurement data, seem to be the effective approaches to reduce the negative impact of measurement uncertainty.

\section{Value of prognostic information in maintenance decision-making}

This section concentrates on quantifying the value of prognostic information in maintenance decisionmaking. The question that arises is: under which conditions can incorporating prognostic information into a maintenance decision be an advantage over a more classical maintenance strategy? From the structures of the considered policies, we can see that the inspection cost $C_{i}$ and system inactivity cost rate $C_{d}$ are the principal elements which cause differences in the maintenance cost savings. So, to answer 
the above question, we vary $C_{i}$ and $C_{d}$, and we investigate the corresponding evolution of the optimal long-run expected maintenance cost rate of both the DR policy and the QIR policy. The comparison of these costs to the costs incurred by the BR policy and the PIR policy assesses the benefit of the dynamic maintenance structure, and justifies or not the choice to invest in prognostic approaches.

\subsection{Numerical results}

Two cases of degradation variance are considered:

1. low variance in deterioration increment with $C=0.015, n=0.35, \beta=3.9$, and $\sigma_{\omega}^{2}=2.53$; and

2. high variance in deterioration increment with $C=0.0047, n=0.35, \beta=3.9$, and $\sigma_{\omega}^{2}=4.8326$.

The average deterioration rates are the same for these cases, and the degradation variance in case 2 is 10 times greater than the one in case 1. The crack depth is revealed through ultrasonic measurement, which is defined by the parameters $\beta_{0}=0.06, \beta_{1}=1.25$, and $\sigma_{v}^{2}=0.0625$. The other parameters are $d=6, \Delta t=1, N_{s}=3000$, and $N_{h}=4000$.

The corrective and preventive replacement costs do not have much influence on the difference between the considered policies, so we fix the preventive replacement $\operatorname{cost} C_{p}=50$, and corrective replacement cost $C_{c}=100$. To analyze the sensitivity of the maintenance policies to the other maintenance costs, the following cases that satisfy the practical constraint $C_{i}<C_{p}<C_{c}$ are studied.

1. Variable inspection cost $C_{i}$ varies from 3 to 19 with unit step 2 ; and fixed costs $C_{p}=50, C_{c}=100$, and $C_{d}=250$.

2. Variable cost rate of system inactivity $C_{d}$ varies from 50 to 350 with unit step 50; and fixed costs $C_{i}=7, C_{p}=50$, and $C_{c}=100$.

The results of these case studies are shown in Figs. 7, and 8.

\subsection{Analysis and discussion on numerical results}

In comparison with the BR policy, Figs. 7 and 8 show clearly that for any value of inspection cost and system inactivity cost rate, the DR policy can always lead to substantial savings. This result can be explained by the fact that the DR policy can adapt its replacement time to the system state, and that it can be regarded as a general case of the BR policy; in the case of very high inspection cost, the BR policy becomes the same as the BR policy. If we compare now the DR policy to the PIR policy, the conclusions are less simple. The performance of the DR policy depends on the maintenance costs, and on the characteristics of the system degradation. When the variance in the degradation increment is quite small, the evolution of the system degradation is more deterministic (in the sense that the evolution 


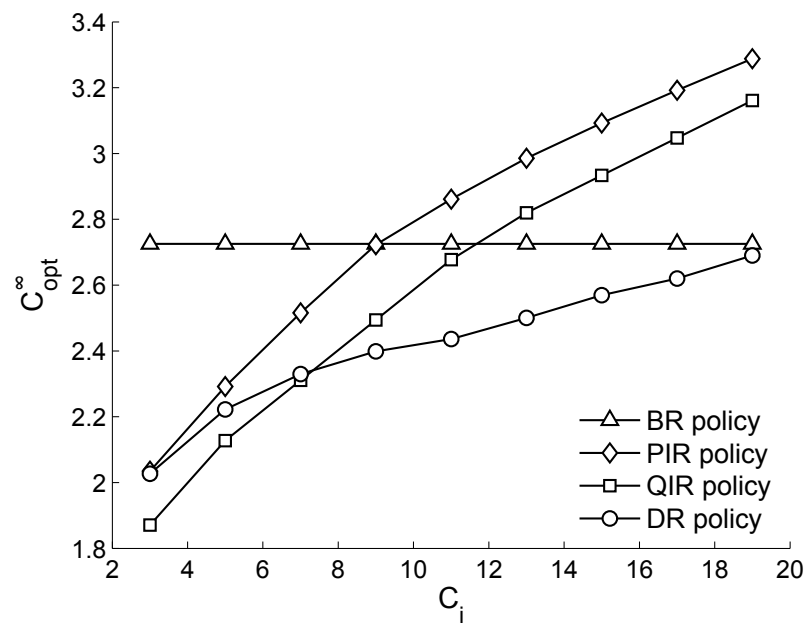

(a) Low variance.

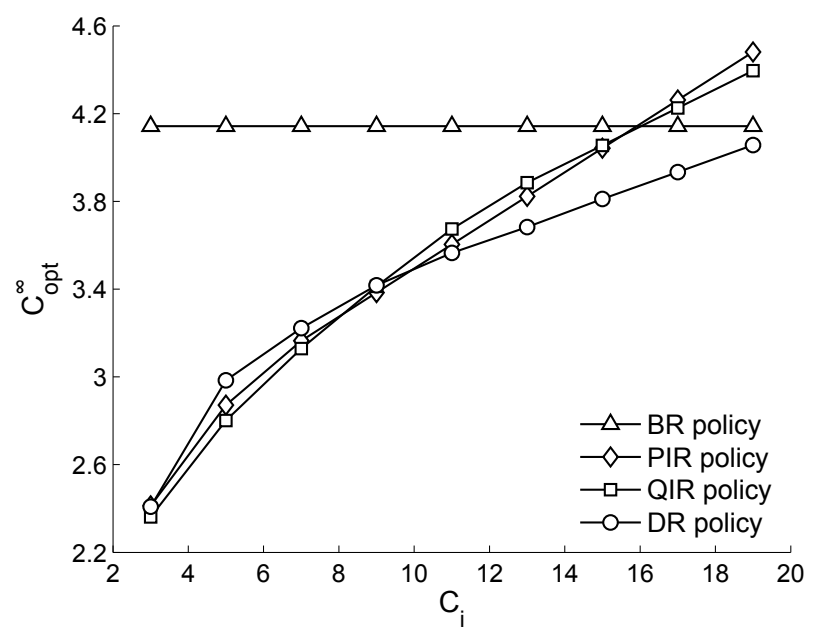

(b) High variance.

Figure 7: Varied inspection cost.

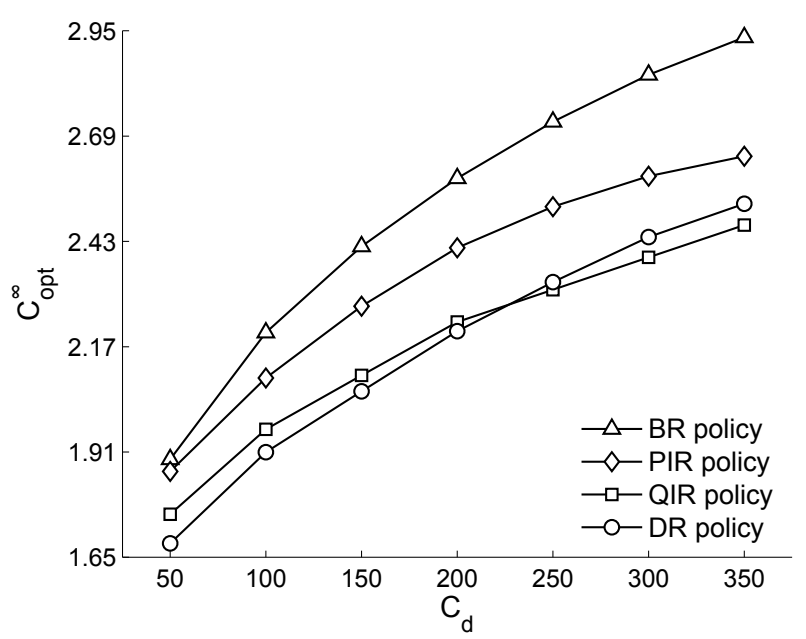

(a) Low variance.

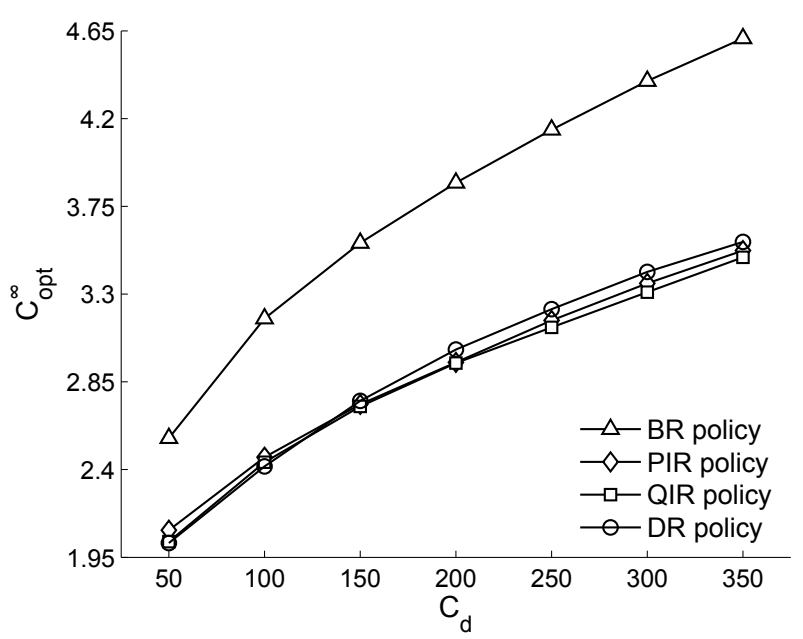

(b) High variance.

Figure 8: Varied cost rate of system inactivity.

of the mean of the degradation is more representative of the actual one), and the inspections become less useful, whereas the same cost $C_{i}$ is incurred. This situation is adequate to perform the DR policy because, at time $T_{\text {pred }}^{(i)}$, no inspection is required to trigger the maintenance (see Fig. 7a). Moreover, because at time $T_{i}$ information returned by inspection is used to adapt the next intervention time $T_{\text {pred }}^{(i)}$, this policy can guarantee a good availability level of the system. Hence, it is better than the PIR policy for cases of variable inactivity cost rate (see Fig. 8a). This result is no longer true when the variance in degradation is higher. The real evolution of the system has a higher probability of being far from the $s$-mean, so the inspections become more useful. Hence, for inspection costs below a given threshold (around 9 in Fig. 7b), the PIR policy is more profitable than the DR policy, and the reverse is true above this threshold. One can note that the DR and the PIR policies are more or less equivalent when the inspection cost $C_{i}=7$ for any value of the inactivity rate (see Fig. 8b). 
Regarding the QIR policy, Fig. 7 shows that it is more profitable than the BR policy when the inspection cost is not too high, as its preventive replacement decision and replacement time can adapt to system states. When the inspection cost increases, the QIR policy becomes less profitable compared to the BR policy, and this result is all the more true if the variance is low. In this case, the QIR decision rules turn into a BR structure, but with a higher cost because an additional and useless inspection cost to estimate the system state is incurred at each time $T_{i}$. When we compare the QIR policy to the PIR policy, a first conclusion is that the dynamic non-periodic inspection scheme in the QIR model yields more savings for any value of the inspection cost and inactivity cost rate. A second very important conclusion is that this trend is especially true as the variance of the degradation process is low (see Figs. 7a, and 8a). This observation clearly shows that the interest of using a dynamic policy with realtime prediction is not at all obvious. If we think about the dynamic structure of the QIR policy, one can first imagine that it must be efficient in the case of high process variance because the mean evolution is not representative, and this policy can adapt in real time the inspection scheme to the real system state. Nevertheless, as shown in Figs. $7 \mathrm{~b}$ and $8 \mathrm{~b}$, the dynamic adaptation in the QIR policy seems to be relatively inefficient in the cases of high variance. Hence, we prefer in this case a periodic-inspection scheme of the PIR type, which is easier to implement.

Concerning the DR and QIR policies, their maintenance cost savings depend on the inspection cost, and the inactivity cost rate. Generally speaking, QIR, which is both condition-based and dynamic, performs better for reasonable inspection costs and high inactivity cost rates, particularly when the variance of the degradation process is low.

To conclude, under different possible characteristics of the system and maintenance costs, both maintenance policies DR and QIR based on prognostic information can lead to more substantial maintenance cost savings than classical policies. However, the present results show that the choice of a strategy is not obvious, and is strongly quantitatively dependent on many parameters such as the maintenance and inspection costs, or the degradation variance. Each configuration needs to be considered, and the numerical studies presented here justify the use of quantification models to make a decision.

\section{Conclusion}

To assess the value of online information, and the impact of measurement uncertainty, we have developed complete maintenance models integrating the characteristics of degradation phenomenon, monitoring performance, the nature of maintenance decision rules, and maintenance and inspection costs. These models are jointly evaluated by an analytical approach (using probability theory), and 
a simulation approach (using the PF technique, and Monte Carlo simulation). Such an evaluation allows us to break out of classical assumptions of maintenance (stationary degradation phenomenon, perfect monitoring information, static maintenance structure, etc.) that can be completely optimized by an analytical approach. Through the considered model, we show that the CBM policy is still more profitable than the TBM policy for a reasonable level of condition monitoring errors. Applying a dynamic maintenance structure, and making a decision based on the estimated degradation constructed from the measurement data, are the potential approaches to reduce the negative impact of measurement uncertainty. Applying on-line prognostics in maintenance decisions can also yield considerable cost savings, and it can be a new orientation for future maintenance strategies to avoid inopportune actions. However, the choice to resort to prognostic approaches, as well as to invest in condition monitoring devices, must be made with caution, and must rely for each configuration on a precise quantification step.

Further research should integrate information on the operating environment of the system to construct a complete degradation and failure model for maintenance decision making.

\section{Acknowledgements}

This work is part of the Ph.D. research work of Khac Tuan HUYNH, financially supported by Conseil Régional de Champagne-Ardernne, France.

[1] A.K.S. Jardine, D. Lin, and D. Banjevic, "A review on machinery diagnostics and prognostics implementing condition-based maintenance," Mechanical Systems and Signal Processing, vol. 20, no. 7 , pp. $1483-1510,2006$.

[2] J.M. Van Noortwijk, "A survey of the application of gamma processes in maintenance," Reliability Engineering \& System Safety, vol. 94, no. 1, pp. 2-21, 2009.

[3] Y. Peng, M. Dong, and M.J. Zuo, "Current status of machine prognostics in condition-based maintenance: a review," The International Journal of Advanced Manufacturing Technology, vol. 50, no. 1-4, pp. 297-313, 2010.

[4] W. Wang, "Overview of a semi-stochastic filtering approach for residual life estimation with applications in condition based maintenance," Proceedings of the Institution of Mechanical Engineers, Part O: Journal of Risk and Reliability, vol. 225, no. 2, pp. 185-197, 2011.

[5] M. Fouladirad, A. Grall, and L. Dieulle, "On the use of on-line detection for maintenance of gradually deteriorating systems," Reliability Engineering \& System Safety, vol. 93, no. 12, pp. 1814$1820,2008$. 
[6] A. Ponchet, M. Fouladirad, and A. Grall, "Assessment of a maintenance model for a multideteriorating mode system," Reliability Engineering 85 System Safety, vol. 95, no. 11, pp. 1244-1254, 2010.

[7] W. Wang, "Condition-based maintenance modelling," in Complex System Maintenance Handbook, Springer Series in Reliability Engineering, pp. 111-131, Springer London, 2008.

[8] A.H. Christer, W. Wang, and J.M. Sharp, "A state space condition monitoring model for furnace erosion prediction and replacement," European Journal of Operational Research, vol. 101, no. 1, pp. 1-14, 1997.

[9] W. Wang and A.H. Christer, "Towards a general condition based maintenance model for a stochastic dynamic system," Journal of the Operational Research Society, vol. 51, no. 2, pp. 145-155, 2000.

[10] M. Rabbani, N. Manavizadeh, and S. Balali, "A stochastic model for indirect condition monitoring using proportional covariate model," International Journal of Engineering Transactions A: Basis, vol. 21, no. 1, pp. 45-56, 2008.

[11] X.S. Si, W. Wang, C.H. Hu, and D.H. Zhou, "Remaining useful life estimation - a review on the statistical data driven approaches," European Journal of Operational Research, vol. 213, no. 1, pp. 1-14, 2011.

[12] A. Grall, L. Dieulle, C. Bérenguer, and M. Roussignol, "Continuous-time predictive-maintenance scheduling for a deteriorating system," IEEE Transactions on Reliability, vol. 51, no. 2, pp. 141-150, 2002.

[13] C. Meier-Hirmer, G. Riboulet, F. Sourget, and M. Roussignol, "Maintenance optimization for a system with a gamma deterioration process and intervention delay: application to track maintenance," Proceedings of the Institution of Mechanical Engineers, Part O: Journal of Risk and Reliability, vol. 223, no. 3, pp. 189-198, 2009.

[14] E. Myötyri, U. Pulkkinen, and K. Simola, "Application of stochastic filtering for lifetime prediction," Reliability Engineering \&3 System Safety, vol. 91, no. 2, pp. 200-208, 2006.

[15] K.T. Huynh, A. Barros, C. Bérenguer, and I.T. Castro, "A periodic inspection and replacement policy for systems subject to competing failure modes due to degradation and traumatic events," Reliability Engineering \& System Safety, vol. 96, no. 4, pp. 497-508, 2011.

[16] F. Cadini and E. Zio, "A Monte Carlo method for the model-based estimation of nuclear reactor dynamics," Annals of Nuclear Energy, vol. 34, no. 10, pp. 773-781, 2007.

[17] F. Cadini and E. Zio, "Application of particle filtering for estimating the dynamics of nuclear systems," IEEE Transactions on Nuclear Science, vol. 55, no. 2, pp. 748-757, 2008. 
[18] F. Cadini, E. Zio, and D. Avram, "Monte Carlo-based filtering for fatigue crack growth estimation," Probabilistic Engineering Mechanics, vol. 24, no. 3, pp. 367-373, 2009.

[19] F. Cadini, E. Zio, and D. Avram, "Model-based Monte Carlo state estimation for condition-based component replacement," Reliability Engineering ES System Safety, vol. 94, no. 3, pp. 752-758, 2009.

[20] M. Marseguerra and E. Zio, "Monte Carlo simulation for model-based fault diagnosis in dynamic systems," Reliability Engineering 8 System Safety, vol. 94, no. 2, pp. 180-186, 2009.

[21] F. Cadini, D. Avram, and E. Zio, "System state estimation by particle filtering for fault diagnosis and prognosis," Proceedings of the Institution of Mechanical Engineers, Part O: Journal of Risk and Reliability, vol. 224, no. 3, pp. 149-158, 2010.

[22] E. Zio and G. Peloni, "Particle filtering prognostic estimation of the remaining useful life of nonlinear components," Reliability Engineering E System Safety, vol. 96, no. 3, pp. 403-409, 2011.

[23] L. Mann, A. Saxena, and G.M. Knapp, "Statistical-based or condition-based preventive maintenance?," Journal of Quality in Maintenance Engineering, vol. 1, no. 1, pp. 46-59, 1995.

[24] F. Kozin and J.L. Bogdanoff, "Probabilistic models of fatigue crack growth: results and speculations," Nuclear Engineering and Design, vol. 115, no. 1, pp. 143-171, 1989.

[25] M. Bigerelle and A. Iost, "Bootstrap analysis of FCGR, application to the Paris relationship and to lifetime prediction," International Journal of Fatigue, vol. 21, no. 4, pp. 299-307, 1999.

[26] I.C. Whittaker and S.C. Saunders, "Application of reliability analysis to aircraft structures subject to fatigue crack growth and periodic structural inspection," Tech. Rep. AFML-TR-73-92, Air Force Material Laboratories, 1973.

[27] Y.K. Lin and J.N. Yang, "On statistical moments of fatigue crack propagation," Engineering Fracture Mechanics, vol. 18, no. 2, pp. 243-256, 1983.

[28] F. Kozin and J.L. Bogdanoff, "A critical analysis of some probabilistic models of fatigue crack growth," Engineering Fracture Mechanics, vol. 14, no. 1, pp. 59-89, 1981.

[29] J.N. Yang and S.D. Manning, "A simple second order approximation for stochastic crack growth analysis," Engineering Fracture Mechanics, vol. 53, no. 5, pp. 677-686, 1996.

[30] J. Xing, O.P. Zhong, and Y.J. Hong, "A simple log normal random process approach of the fatigue crack growth considering the distribution of initial crack size and loading condition," International Journal of Pressure Vessels and Piping, vol. 74, no. 1, pp. 7-12, 1997.

[31] W.F. Wu and C.C. Ni, "A study of stochastic fatigue crack growth modeling through experimental data," Probabilistic Engineering Mechanics, vol. 18, no. 2, pp. 107-118, 2003.

[32] W. Wu and C. Ni, "Probabilistic models of fatigue crack propagation and their experimental veri- 
fication," Probabilistic Engineering Mechanics, vol. 19, no. 3, pp. 247-257, 2004.

[33] K. Simola and U. Pulkkinen, "Models for non-destructive inspection data," Reliability Engineering 83 System Safety, vol. 60, no. 1, pp. 1-12, 1998.

[34] H.C. Tijms, A first course in stochastic models. Wiley, New York, 2003.

[35] W. Wang, "Modelling condition monitoring intervals: a hybrid of simulation and analytical approaches," Journal of the Operational Research Society, vol. 54, no. 3, pp. 273-282, 2003.

[36] L. Cui, M. Xie, and H.T. Loh, "Inspection schemes for general systems," IIE Transactions, vol. 36, no. 9 , pp. $817-825,2004$.

[37] Y. Yang and G.A. Klutke, "Improved inspection schemes for deteriorating equipment," Probability in the Engineering and Informational Sciences, vol. 14, no. 4, pp. 445-460, 2000.

[38] M.E. Orchard and G.A. Vachtsevanos, "A particle-filtering approach for on-line fault diagnosis and failure prognosis," Transactions of the Institute of Measurement E Control, vol. 31, no. 3-4, p. 221, 2009.

[39] Y. Sun, L. Ma, J. Mathew, W. Wang, and S. Zhang, "Mechanical systems hazard estimation using condition monitoring," Mechanical Systems and Signal Processing, vol. 20, no. 5, pp. 1189-1201, 2006.

[40] P. Baruah and R.B. Chinnam, "HMMs for diagnostics and prognostics in machining processes," International Journal of Production Research, vol. 43, no. 6, pp. 1275-1293, 2005.

[41] M.S. Arulampalam, S. Maskell, N. Gordon, and T. Clapp, "A tutorial on particle filters for online nonlinear/non-gaussian bayesian tracking," IEEE Transactions on Signal Processing, vol. 50, no. 2, pp. 174-188, 2002.

[42] A. Doucet, N. De Freitas, and N. Gordon, Sequential Monte Carlo methods in practice. Springer Verlag, 2001.

[43] M.Z. Chen, D.H. Zhou, and G.P. Liu, "A new particle predictor for fault prediction of nonlinear time-varying systems," Developments in Chemical Engineering and Mineral Processing, vol. 13, no. 3-4, pp. 379-388, 2005.

[44] Z. Xu, Y. Ji, and D. Zhou, "A new real-time reliability prediction method for dynamic systems based on on-line fault prediction," IEEE Transactions on Reliability, vol. 58, no. 3, pp. 523-538, 2009.

[45] G. Kitagawa, "Monte carlo filter and smoother for non-gaussian nonlinear state space models," Journal of Computational and Graphical Statistics, vol. 5, no. 1, pp. 1-25, 1996. 
Khac Tuan Huynh is a lecturer at Troyes University of Technology (UTT), France, and is a member of the Systems Modeling and Dependability Laboratory. He received the M.S., and Ph.D. degrees in System Safety and Optimization from the UTT in 2008, and 2011 respectively. One of his papers has received First Place in the 2010 Tom Fagan Reliability \& Maintainability Symposium Student Paper Competition. His current research interests include stochastic modeling of systems deterioration, and maintenance planning, optimization and evaluation.

Anne Barros is a professor at the Troyes University of Technology (UTT), and Head of the System Safety and Optimization Ph.D. program at the University of Technology of Troyes (France). Her research interests focus on the construction of probabilistic decision indicators used for safety assessment, and maintenance optimization. These indicators are based on stochastic modeling of the system state, its environment, and generally speaking of the whole information given by the monitoring device. She has authored papers on stochastic models in reliability or maintenance optimization in IEEE Transactions on Reliability, Reliability Engineering and System Safety, Journal of Risk and Reliability, Journal of Loss Prevention in the Process Industries, International Journal of reliability, and Quality and Safety Engineering.

Christophe Bérenguer is a professor of reliability engineering and control systems at Gipsa-Lab and Grenoble Institute of Technology (Grenoble, France). From 1997 to 2011, he was a professor at Troyes University of Technology (Troyes, France). He has served as an officer (treasurer) for the European Safety and Reliability Association from 2005 to 2010. He is a member of the Editorial Boards of Reliability Engineering and System Safety, and Journal of Risk and Reliability. His research interests include system health monitoring, stochastic modeling of systems deterioration, performance evaluation and optimization of dynamic maintenance policies, and probabilistic safety assessment. 